BI-TP 2010/16

\title{
Colour-electric spectral function at next-to-leading order
}

\author{
Y. Burnier, M. Laine, J. Langelage, L. Mether \\ Faculty of Physics, University of Bielefeld, D-33501 Bielefeld, Germany
}

\begin{abstract}
The spectral function related to the correlator of two colour-electric fields along a Polyakov loop determines the momentum diffusion coefficient of a heavy quark near rest with respect to a heat bath. We compute this spectral function at next-to-leading order, $\mathcal{O}\left(\alpha_{s}^{2}\right)$, in the weakcoupling expansion. The high-frequency part of our result $(\omega \gg T)$, which is shown to be temperature-independent, is accurately determined thanks to asymptotic freedom; the lowfrequency part of our result $(\omega \ll T)$, in which Hard Thermal Loop resummation is needed in order to cure infrared divergences, agrees with a previously determined expression. Our result may help to calibrate the overall normalization of a lattice-extracted spectral function in a perturbative frequency domain $T \ll \omega \ll 1 / a$, paving the way for a non-perturbative estimate of the momentum diffusion coefficient at $\omega \rightarrow 0$. We also evaluate the colour-electric Euclidean correlator, which could be directly compared with lattice simulations. As an aside we determine the Euclidean correlator in the lattice strong-coupling expansion, showing that through a limiting procedure it can in principle be defined also in the confined phase of pure Yang-Mills theory, even if a practical measurement could be very noisy there.
\end{abstract}

June 2010 


\section{Introduction}

In order to theoretically model various observations made at relativistic heavy ion collision experiments, it would be important to quantitatively determine a number of "transport coefficients" of Quantum Chromodynamics (QCD) at temperatures around a few hundred MeV. Such transport coefficients include for instance the shear and bulk viscosities and the thermal conductivity, which play a role in the hydrodynamic equations describing the expansion of the thermal fireball; as well as heavy flavour diffusion coefficients, the jet quenching parameter, and the electrical conductivity, which dictate how various "probes" propagate with respect to the hydrodynamically expanding thermal medium (for recent reviews, see refs. [1, 2]).

Among all the transport coefficients, the theoretically most accessible one appears to be the one related to the diffusive motion of heavy quarks (charm or bottom) near rest with respect to the thermal medium. In a classical Langevin picture, the heavy quarks satisfy the equation of motion $\mathrm{d} p_{i} / \mathrm{d} t=-\eta_{\mathrm{D}} p_{i}+\xi_{i}$, where $p_{i}$ is their momentum and $\xi_{i}$ is a random force; the transport coefficient $\eta_{\mathrm{D}}$ can be referred to as the "kinetic thermalization rate" or the "drag coefficient". The simplicity of its determination is due to the fact that it can be fluctuation-dissipation related to another coefficient, $\kappa=\frac{1}{3} \int_{-\infty}^{\infty} \sum_{i}\left\langle\xi_{i}(t) \xi_{i}(0)\right\rangle$, called the "momentum diffusion coefficient" (the relation reads $\eta_{\mathrm{D}}=\kappa /\left(2 T M_{\text {kin }}\right)\left(1+\mathcal{O}\left(T / M_{\text {kin }}\right)\right)$, where $M_{\text {kin }}$ is a certain heavy quark mass definition); $\xi_{i}$ can in turn be identified as the Lorentz force, which for small velocities is proportional to the electric field strength. Thereby $\kappa$ can be determined from the two-point correlator of colour-electric fields along a timelike Wilson line [3, 4]. The correlator of colour-electric fields is simpler to handle theoretically than that of the usual bilinear quark currents, because fewer physical scales enter the problem (heavy quark related scales like $M_{\text {kin }}$ do not appear) and because no transport peak is present.

Within this framework, a number of results have recently been obtained. First of all, the leading perturbative expression for $\kappa[5]-7]$ has been supplemented by a next-to-leading order correction [8], which was furthermore shown to be so large as to question the validity of the weak-coupling expansion. (This computation can be contrasted with the corresponding results for theories with gravity duals, in particular for strongly-coupled $\mathcal{N}=4$ Yang-Mills theory in the large- $N_{\mathrm{c}}$ limit [9, 3], in which case $\kappa$ appears to be much "larger" than according to leading-order QCD.) Second, the frequency dependence of the correlator $\kappa(\omega)=\frac{1}{3} \int_{-\infty}^{\infty} e^{i \omega t} \sum_{i}\left\langle\xi_{i}(t) \xi_{i}(0)\right\rangle$ has been determined within various frameworks, such as strongly coupled $\mathcal{N}=4$ Yang-Mills theory in the large- $N_{\mathrm{c}}$ limit [3, 10, Hard Thermal Loop resummed perturbation theory [4, as well as classical lattice gauge theory [11]. Gaining understanding on the frequency dependence is important because a non-perturbative extraction of $\kappa$ from lattice QCD would effectively necessitate an ansatz for the shape of $\kappa(\omega)$.

Apart from these theoretical developments, heavy quark diffusion has also been embedded in phenomenological models (see, e.g., refs. [7, 12] and references therein). Comparing with 
experimental data [13], it appears that the physical value of $\kappa$ would need to be significantly larger than the leading-order [5]-[7] or even the next-to-leading order one [8, perhaps in qualitative accord with the AdS/CFT result [9, 3] (cf. also ref. [14] and references therein).

The purpose of this paper is to address the frequency dependence of $\kappa(\omega)$ within QCD and pure Yang-Mills theory, with an eventual lattice determination of $\kappa \equiv \kappa(0)$ in mind. To this end, we determine $\kappa(\omega)$ at next-to-leading order, $\mathcal{O}\left(\alpha_{s}^{2}\right)$, in the weak-coupling expansion. Thanks to asymptotic freedom, our result becomes increasingly accurate in the large-frequency limit where, as we show, the result is also independent of the temperature. In fact, in several respects, the result at $\omega \gtrsim T$ is analogous to that obtained a long time ago for the spectral function related to the electromagnetic current [15]; nevertheless, we are not aware of a previous computation of a spectral function at $\mathcal{O}\left(\alpha_{s}^{2}\right)$ consistently applicable in the whole frequency range from $\omega \lesssim \alpha_{s}^{1 / 2} T$ up to $\omega \gg T$.

The paper is organized as follows. In section 2 we define the basic observable considered and introduce our notation. The main steps of the computation are outlined and to some extent also carried out in section 3, a number of technical details are relegated to two appendices. Section 4 collects together various results, both in analytic form as well as numerically. Section 5 is complementary to the main body of this work, addressing the colour-electric field correlator in the lattice strong-coupling expansion. We summarize and offer an outlook in section 6 .

\section{Basic definitions}

The computation is carried out within continuum QCD, with $N_{\mathrm{c}}$ colours and $N_{\mathrm{f}}$ massless quark flavours. The theory is regularized dimensionally, and renormalized quantities are expressed in the $\overline{\mathrm{MS}}$ scheme. The only parameter requiring renormalization is the gauge coupling; the bare gauge coupling is denoted by $g_{\mathrm{B}}$, the renormalized one by $g$, and $\alpha_{s} \equiv$ $g^{2} / 4 \pi$. The relation between $g_{\text {В }}$ and $g$ will only be needed at 1-loop order,

$$
g_{\mathrm{B}}^{2}=g^{2}+\frac{g^{4} \mu^{-2 \epsilon}}{(4 \pi)^{2}} \frac{2 N_{\mathrm{f}}-11 N_{\mathrm{c}}}{3 \epsilon}+\mathcal{O}\left(g^{6}\right),
$$

where $\mu$ is a scale parameter related to "minimal subtraction", and the corresponding $\overline{\mathrm{MS}}$ scheme quantity is denoted by $\bar{\mu}^{2}=4 \pi e^{-\gamma_{\mathrm{E}}} \mu^{2}$. The factor $\mu^{-2 \epsilon}$ is normally not displayed explicitly; the same holds for corrections of $\mathcal{O}(\epsilon)$ which are not multiplied by divergent coefficients. A group theory factor appearing frequently is defined by $C_{F} \equiv\left(N_{\mathrm{c}}^{2}-1\right) / 2 N_{\mathrm{c}}$.

The observable considered contains colour-electric fields and temporal Wilson lines. Employing sign conventions where the covariant derivative reads $D_{\mu}=\partial_{\mu}-i g_{\mathrm{B}} A_{\mu}$, with $A_{\mu}$ a traceless and hermitean gauge field, we define a temporal Wilson line at spatial position 
$\mathbf{r} \equiv \mathbf{0}$ through

$$
U\left(\tau_{b}, \tau_{a}\right) \equiv \mathbb{1}+i g_{\mathrm{B}} \int_{\tau_{a}}^{\tau_{b}} \mathrm{~d} \tau A_{0}(\tau, \mathbf{0})+\left(i g_{\mathrm{B}}\right)^{2} \int_{\tau_{a}}^{\tau_{b}} \mathrm{~d} \tau \int_{\tau_{a}}^{\tau} \mathrm{d} \tau^{\prime} A_{0}(\tau, \mathbf{0}) A_{0}\left(\tau^{\prime}, \mathbf{0}\right)+\ldots
$$

A colour-electric field strength is defined through

$$
g_{\mathrm{B}} E_{i} \equiv i\left[D_{0}, D_{i}\right]
$$

With this notation, the Euclidean correlation function considered [4] can be expressed as

$$
G_{\mathrm{E}}(\tau) \equiv-\frac{1}{3} \sum_{i=1}^{3-2 \epsilon} \frac{\left\langle\operatorname{Re} \operatorname{Tr}\left[U(\beta, \tau) g_{\mathrm{B}} E_{i}(\tau, \mathbf{0}) U(\tau, 0) g_{\mathrm{B}} E_{i}(0, \mathbf{0})\right]\right\rangle}{\langle\operatorname{Re} \operatorname{Tr}[U(\beta, 0)]\rangle}
$$

where $\beta \equiv 1 / T$ is the inverse temperature. Note that we keep a fixed " 3 " in the denominator even in the presence of dimensional regularization; this has no effect on the final renormalized result but does affect the finite parts of divergent intermediate expressions.

The spectral function corresponding to $G_{\mathrm{E}}(\tau)$ can be determined from

$$
\begin{aligned}
\tilde{G}_{\mathrm{E}}\left(\omega_{n}\right) & \equiv \int_{0}^{\beta} \mathrm{d} \tau e^{i \omega_{n} \tau} G_{\mathrm{E}}(\tau), \\
\rho_{\mathrm{E}}(\omega) & =\operatorname{Im} \tilde{G}_{\mathrm{E}}\left(\omega_{n} \rightarrow-i\left[\omega+i 0^{+}\right]\right),
\end{aligned}
$$

where the analytic continuation becomes unique by requiring powerlike (not exponential) growth at asymptotic frequencies; the momentum diffusion coefficient then follows from

$$
\kappa \equiv \lim _{\omega \rightarrow 0} \frac{2 T \rho_{\mathrm{E}}(\omega)}{\omega}
$$

If only a numerical determination of $G_{\mathrm{E}}(\tau)$ is available, the task would be to invert the relation

$$
G_{\mathrm{E}}(\tau)=\int_{0}^{\infty} \frac{\mathrm{d} \omega}{\pi} \rho_{\mathrm{E}}(\omega) \frac{\cosh \left(\frac{\beta}{2}-\tau\right) \omega}{\sinh \frac{\beta \omega}{2}}
$$

for which various practical recipes have been proposed; see, e.g., refs. [16]. (Probably the asymptotic large- $\omega$ behaviour of $\rho_{\mathrm{E}}(\omega)$ is needed as input for the inversion.)

The graphs contributing to the numerator of $G_{\mathrm{E}}$ are shown up to $\mathcal{O}\left(g^{4}\right)$ in fig. 1. As is typically the case in finite-temperature QCD, however, certain subclasses of higher-order graphs may need to be considered as well. This can be achieved with the so-called Hard Thermal Loop (HTL) effective theory [17]; where necessary, the observables will then be expressed as

$$
G_{\mathrm{E}}=\left[\left(G_{\mathrm{E}}\right)_{\mathrm{QCD}}-\left(G_{\mathrm{E}}\right)_{\mathrm{HTL}}\right]_{\text {naive }}+\left[\left(G_{\mathrm{E}}\right)_{\mathrm{HTL}}\right]_{\text {resum }},
$$




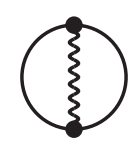

(a)

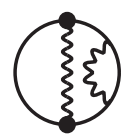

(b)

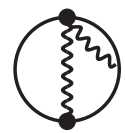

(g)

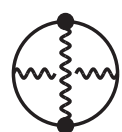

(c)

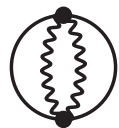

(h)

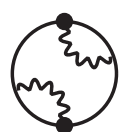

(d)

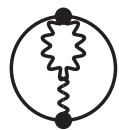

(i)

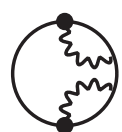

(e)

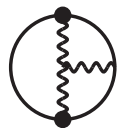

(j)

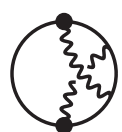

(f)

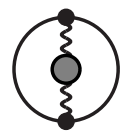

(k)

Figure 1: The graphs contributing to the colour-electric field correlator, $G_{\mathrm{E}}(\tau)$ defined in eq. (2.4), up to $\mathcal{O}\left(g^{4}\right)$. The big circle denotes a Wilson line wrapping around the time direction; the small dots the colour-electric field strengths; and the grey blob the 1-loop gauge field self-energy. Graphs obtained with trivial "reflections" from those shown have been omitted from the figure.

where "naive" refers to an unresummed computation and "resum" to a resummed one. The unresummed difference in the first square brackets is infrared finite (provided that the correct low-energy effective description is used), and can be computed in naive perturbation theory, i.e. from the graphs of fig. 1; in an effective field theory language, it corresponds to an ultraviolet matching coefficient. The last term of eq. (2.9) is infrared sensitive, but can be addressed within the simplified effective description rather than with full QCD.

\section{Outline of the computation}

\subsection{Leading order analysis}

In order to illustrate the steps of the computation, we start by explicitly deriving the (wellknown) leading order result for the spectral function, corresponding to the contribution of graph (a) of fig. 1. A direct evaluation of the Wick contractions leads to

$$
G_{\mathrm{E}}^{(2)}(\tau)=\frac{g_{\mathrm{B}}^{2} C_{F}}{3} \sum_{i}\left\{\partial_{0}^{2} \Delta_{i i}(\tau, \mathbf{0})+\partial_{i}^{2} \Delta_{00}(\tau, \mathbf{0})-\partial_{0} \partial_{i}\left[\Delta_{0 i}(\tau, \mathbf{0})+\Delta_{i 0}(\tau, \mathbf{0})\right]\right\}
$$

where $\Delta_{\mu \nu}$ is the gauge field propagator in configuration space. Expressing the propagator in momentum space,

$$
\Delta_{\mu \nu}(\tau, \mathbf{x})=\oiint_{K} e^{i k_{n} \tau+i \mathbf{k} \cdot \mathbf{x}} \tilde{\Delta}_{\mu \nu}(K),
$$

where $\mathscr{\&}_{K} \equiv T \sum_{k_{n}} \int_{\mathbf{k}}$ and $k_{n}$ is a bosonic Matsubara frequency, $k_{n}=2 \pi T n, n \in \mathbb{Z}$; and inserting the propagator of the general covariant gauge,

$$
\tilde{\Delta}_{\mu \nu}(K)=\frac{\delta_{\mu \nu}}{K^{2}}+\frac{\tilde{\xi} K_{\mu} K_{\nu}}{\left(K^{2}\right)^{2}},
$$


the longitudinal part proportional to the gauge parameter, $\tilde{\xi}$, is seen to drop out. The remaining expression reads

$$
G_{\mathrm{E}}^{(2)}(\tau)=-\frac{g_{\mathrm{B}}^{2} C_{F}}{3} \sum_{K} e^{i k_{n} \tau} \frac{(D-1) k_{n}^{2}+\mathbf{k}^{2}}{K^{2}},
$$

where $D=4-2 \epsilon$ is the spacetime dimensionality. Writing $k_{n}^{2}=K^{2}-\mathbf{k}^{2}$, omitting the scaleless integral $\int_{\mathbf{k}} 1$ due to properties of dimensional regularization, and making use of

$$
T \sum_{k_{n}} \frac{e^{i k_{n} \tau}}{k_{n}^{2}+k^{2}}=\frac{n_{\mathrm{B}}(k)}{2 k}\left[e^{(\beta-\tau) k}+e^{\tau k}\right], \quad 0 \leq \tau \leq \beta,
$$

where $k \equiv|\mathbf{k}|$, we get

$$
G_{\mathrm{E}}^{(2)}(\tau)=\frac{g_{\mathrm{B}}^{2} C_{F}(D-2)}{6} \int_{\mathbf{k}} k n_{\mathrm{B}}(k)\left[e^{(\beta-\tau) k}+e^{\tau k}\right] .
$$

The Fourier transform as in eq. (2.5) yields

$$
\tilde{G}_{\mathrm{E}}^{(2)}\left(\omega_{n}\right)=\frac{g_{\mathrm{B}}^{2} C_{F}(D-2)}{6} \int_{\mathbf{k}} k\left[\frac{1}{k-i \omega_{n}}+\frac{1}{k+i \omega_{n}}\right]
$$

and taking a subsequent discontinuity according to eq. (2.6) we obtain

$$
\rho_{\mathrm{E}}^{(2)}(\omega)=\frac{g_{\mathrm{B}}^{2} C_{F}(D-2) \pi}{6} \int_{\mathbf{k}} k[\delta(k-\omega)-\delta(k+\omega)] .
$$

The final integral over $\mathbf{k}$ can now be trivially carried out. For $D=4$ the result reads

$$
\rho_{\mathrm{E}}^{(2)}(\omega)=\frac{g^{2} C_{F}}{6 \pi} \omega^{3}
$$

where we also expanded the bare gauge coupling according to eq. (2.1).

\subsection{Wick contractions at next-to-leading order}

Proceeding to the level $\mathcal{O}\left(g^{4}\right)$, the structures in the graphs (b)-(k) of fig. 1 need to be considered. (There is also a disconnected contribution originating from the numerator of eq. (2.4), as will be specified presently.) Given that the observable is gauge invariant, the full result must be independent of the gauge parameter $\tilde{\xi}$; nevertheless the individual diagrams contain non-trivial terms proportional to $\tilde{\xi}$ and $\tilde{\xi}^{2}$. We have checked explicitly that both structures cancel in the sum, and display intermediate expressions only for the Feynman gauge, $\tilde{\xi}=0$. (As a further cross-check, the small-frequency limit will be compared against that of an independent computation in the Coulomb gauge, cf. sec. 4.3). 
Considering first the structures (b)-(f) of fig. 1, and combining them with the contribution of the denominator of eq. (2.4), viz.

$$
\langle\operatorname{Re} \operatorname{Tr}[U(\beta, 0)]\rangle=N_{\mathrm{c}}\left[1-g_{\mathrm{B}}^{2} C_{F} \int_{0}^{\beta} \mathrm{d} \tau \int_{0}^{\tau} \mathrm{d} \tau^{\prime} \Delta_{00}\left(\tau^{\prime}, \mathbf{0}\right)\right]+\mathcal{O}\left(g_{\mathrm{B}}^{4}\right),
$$

we obtain

$$
\begin{aligned}
\delta_{(\mathrm{b}-\mathrm{f})} G_{\mathrm{E}}^{(4)}(\tau) & =\frac{g_{\mathrm{B}}^{4} C_{F} N_{\mathrm{c}}}{6}\left[(D-1) \partial_{\tau}^{2}+\nabla^{2}\right] G(\tau, \mathbf{0}) \int_{\tau}^{\beta} \mathrm{d} \tau^{\prime} \int_{0}^{\tau} \mathrm{d} \tau^{\prime \prime} G\left(\tau^{\prime}-\tau^{\prime \prime}, \mathbf{0}\right) \\
& =\frac{g_{\mathrm{B}}^{4} C_{F} N_{\mathrm{c}}}{3} \oiint_{K} \frac{e^{i k_{n} \tau}}{K^{2}}\left[-(D-1) K^{2}+(D-2) k^{2}\right] \oiint_{Q^{\prime}} \frac{e^{i q_{n} \tau}-1}{q_{n}^{2} Q^{2}} .
\end{aligned}
$$

Here we denoted $G\left(x_{0}, \mathbf{x}\right) \equiv\left[\Delta_{00}\left(x_{0}, \mathbf{x}\right)\right]_{\tilde{\xi}=0}$, i.e.

$$
G\left(x_{0}, \mathbf{x}\right)=\sum_{K} \frac{e^{i k_{n} x_{0}+i \mathbf{k} \cdot \mathbf{x}}}{k_{n}^{2}+\mathbf{k}^{2}}
$$

and moved to momentum space, making use of

$$
\int_{0}^{\tau} \mathrm{d} \tau^{\prime} e^{i q_{n} \tau^{\prime}}=\delta_{q_{n}} \tau+\left(1-\delta_{q_{n}}\right) \frac{1}{i q_{n}}\left(e^{i q_{n} \tau}-1\right)
$$

etc, where $q_{n}$ is a bosonic Matsubara frequency and $\delta_{q_{n}} \equiv \delta_{n, 0}$ is a (periodic) Kronecker delta function. It can be verified that the Matsubara zero-mode part of eq. (3.13) does not contribute in dimensional regularization in eq. (3.11); the prime in $\mathbb{W}_{Q^{\prime}}$ indicates that it is omitted. The term with $(D-1) K^{2}$ in eq. (3.11) does not contribute either, because the scaleless integral $\int_{\mathbf{k}} 1$ vanishes in dimensional regularization.

The contributions of the graphs (g) and (h) of fig. 1 can be worked out in a similar way. A straightforward computation yields

$$
\begin{aligned}
\delta_{(\mathrm{g})} G_{\mathrm{E}}^{(4)}(\tau) & =\frac{g_{\mathrm{B}}^{4} C_{F} N_{\mathrm{c}}(D-1)}{3} \partial_{\tau} G(\tau, \mathbf{0}) \int_{\tau}^{\beta-\tau} \mathrm{d} \tau^{\prime} G\left(\tau^{\prime}, \mathbf{0}\right) \\
& =-\frac{2 g_{\mathrm{B}}^{4} C_{F} N_{\mathrm{c}}(D-1)}{3} \sum_{K} \frac{i k_{n} e^{i k_{n} \tau}}{K^{2}} \sum_{Q^{\prime}} \frac{e^{i q_{n} \tau}-1}{i q_{n} Q^{2}}, \\
\delta_{(\mathrm{h})} G_{\mathrm{E}}^{(4)}(\tau) & =-\frac{g_{\mathrm{B}}^{4} C_{F} N_{\mathrm{c}}(D-1)}{3}[G(\tau, \mathbf{0})]^{2} \\
& =-\frac{g_{\mathrm{B}}^{4} C_{F} N_{\mathrm{c}}(D-1)}{3} \sum_{K, Q} \frac{e^{i k_{n} \tau}}{Q^{2}(K-Q)^{2}} .
\end{aligned}
$$

The graphs (i) and particularly (j) of fig. 1 are somewhat more complicated than the ones discussed so far. For (i) a few steps lead to the momentum space expression

$$
\delta_{(\mathrm{i})} G_{\mathrm{E}}^{(4)}(\tau)=g_{\mathrm{B}}^{4} C_{F} N_{\mathrm{c}} \mathcal{F}_{K} e^{i k_{n} \tau} \oint_{Q}\left[\frac{D-1}{Q^{2}(K-Q)^{2}}-\frac{(D-2) k^{2}}{K^{2} Q^{2}(K-Q)^{2}}\right],
$$


while for $(\mathrm{j})$ it is perhaps simplest to remain in configuration space:

$$
\delta_{(\mathrm{j})} G_{\mathrm{E}}^{(4)}(\tau)=-\delta_{(\mathrm{g})} G_{\mathrm{E}}^{(4)}(\tau)+\frac{g_{\mathrm{B}}^{4} C_{F} N_{\mathrm{c}}}{6} \mathcal{I}_{5}(\tau) .
$$

Here we carried out a partial integration and made use of $\left(\partial_{0}^{2}+\nabla^{2}\right) G\left(x_{0}, \mathbf{x}\right)=-\delta^{(4)}(x)$, in order to identify a term that cancels against that in eq. (3.14); and expressed the remainder through the integral

$$
\begin{aligned}
\mathcal{I}_{5}(\tau) & \equiv \int_{x}\left[\int_{\tau}^{\beta} \mathrm{d} \tau^{\prime}-\int_{0}^{\tau} \mathrm{d} \tau^{\prime}\right]\{ \\
& \partial_{i} G\left(x_{0}-\tau^{\prime}, \mathbf{x}\right)\left[\partial_{0} G\left(x_{0}-\tau, \mathbf{x}\right) \partial_{i} G\left(x_{0}, \mathbf{x}\right)-\partial_{i} G\left(x_{0}-\tau, \mathbf{x}\right) \partial_{0} G\left(x_{0}, \mathbf{x}\right)\right] \\
+ & \left.(D-2) G\left(x_{0}-\tau^{\prime}, \mathbf{x}\right)\left[\partial_{0} G\left(x_{0}-\tau, \mathbf{x}\right) \nabla^{2} G\left(x_{0}, \mathbf{x}\right)-\nabla^{2} G\left(x_{0}-\tau, \mathbf{x}\right) \partial_{0} G\left(x_{0}, \mathbf{x}\right)\right]\right\} .
\end{aligned}
$$

The subscript " 5 " refers to the fact that this is the most complicated among the 5 independent structures that appear in our result (the other ones are defined in eqs. (3.25)-(3.28) ) 1 A corresponding momentum space expression, albeit with no care taken of the Matsubara zero mode contribution, is given in eq. (A.30) of appendix A.

Inspecting finally the self-energy contribution, graph $(\mathrm{k})$ in fig. 1, we recall that making use of the substitution $Q \rightarrow K-Q$ in order to simplify the numerator, the Feynman gauge self-energy has the form

$$
\begin{aligned}
\Pi_{\mu \nu}(K) & =\frac{g_{\mathrm{B}}^{2} N_{\mathrm{c}}}{2} \oint_{Q} \frac{\delta_{\mu \nu}\left[-4 K^{2}+2(D-2) Q^{2}\right]+(D+2) K_{\mu} K_{\nu}-4(D-2) Q_{\mu} Q_{\nu}}{Q^{2}(K-Q)^{2}} \\
& -g_{\mathrm{B}}^{2} N_{\mathrm{f}} \oint_{\{Q\}} \frac{\delta_{\mu \nu}\left[-K^{2}+2 Q^{2}\right]+2 K_{\mu} K_{\nu}-4 Q_{\mu} Q_{\nu}}{Q^{2}(K-Q)^{2}},
\end{aligned}
$$

and the corresponding contribution to $G_{\mathrm{E}}(\tau)$ reads

$$
\delta_{(\mathrm{k})} G_{\mathrm{E}}^{(4)}(\tau)=\frac{g_{\mathrm{B}}^{2} C_{F}}{3} \sum_{K} \frac{e^{i k_{n} \tau}}{\left(K^{2}\right)^{2}} \sum_{i}\left(k_{n} \delta_{i \mu}-k_{i} \delta_{0 \mu}\right)\left(k_{n} \delta_{i \nu}-k_{i} \delta_{0 \nu}\right) \Pi_{\mu \nu}(K) .
$$

Following the usual convention, $\mathbb{E}_{\{Q\}}$ in eq. (3.19) means that the Matsubara frequency is fermionic, i.e. $\left\{q_{n}\right\}=(2 n+1) \pi T$, with $n \in \mathbb{Z}$; also, in eq. (3.20), $K=\left(k_{n}, k_{i}\right)$.

Combining eqs. (3.19) and (3.20), the transverse projectors eliminate the parts proportional to $K_{\mu} K_{\nu}$ from $\Pi_{\mu \nu}$, while the parts proportional to $Q_{\mu} Q_{\nu}$ can be expressed as

$$
\sum_{i}\left(k_{n} \delta_{i \mu}-k_{i} \delta_{0 \mu}\right)\left(k_{n} \delta_{i \nu}-k_{i} \delta_{0 \nu}\right) Q_{\mu} Q_{\nu}=(K-Q)^{2} k_{n} q_{n}+Q^{2} k_{n}\left(k_{n}-q_{n}\right)+K^{2} q_{n}\left(q_{n}-k_{n}\right) .
$$

\footnotetext{
${ }^{1}$ In fact $\mathcal{I}_{5}$ could be subdivided into further independent structures, but here we keep it as one entity.
} 
The first two terms are odd in the summation variable $q_{n}$ or $k_{n}-q_{n}$, respectively, and give no contribution in eq. (3.20), so that only the last term matters. In addition there is a contribution from the term proportional to $\delta_{\mu \nu}$ in $\Pi_{\mu \nu}$ which we write as

$$
\sum_{i}\left(k_{n} \delta_{i \mu}-k_{i} \delta_{0 \mu}\right)\left(k_{n} \delta_{i \nu}-k_{i} \delta_{0 \nu}\right) \delta_{\mu \nu}=(D-1) K^{2}-(D-2) k^{2}
$$

In total, then,

$$
\begin{aligned}
& \delta_{(\mathrm{k})} G_{\mathrm{E}}^{(4)}(\tau)=\frac{g_{\mathrm{B}}^{4} C_{F}}{3} \sum_{K} e^{i k_{n} \tau} \\
& \times\left\{\frac{N_{\mathrm{c}}}{2} \sum_{Q}[\right.-\frac{4(D-1)}{Q^{2}(K-Q)^{2}}+\frac{4(D-2) k^{2}}{K^{2} Q^{2}(K-Q)^{2}}-\frac{4(D-2) q_{n}\left(q_{n}-k_{n}\right)}{K^{2} Q^{2}(K-Q)^{2}} \\
&\left.\quad+\frac{2(D-2)}{Q^{2}}\left(\frac{D-1}{K^{2}}-\frac{(D-2) k^{2}}{\left(K^{2}\right)^{2}}\right)\right] \\
&-N_{\mathrm{f}} \sum_{\{Q\}}\left[-\frac{(D-1)}{Q^{2}(K-Q)^{2}}+\frac{(D-2) k^{2}}{K^{2} Q^{2}(K-Q)^{2}}-\frac{4 q_{n}\left(q_{n}-k_{n}\right)}{K^{2} Q^{2}(K-Q)^{2}}\right. \\
&\left.\left.\quad+\frac{2}{Q^{2}}\left(\frac{D-1}{K^{2}}-\frac{(D-2) k^{2}}{\left(K^{2}\right)^{2}}\right)\right]\right\} .
\end{aligned}
$$

We now sum together eqs. (3.11), (3.14)-(3.17), (3.23). It can be noted that, in dimensional regularization,

$$
\int_{\mathbf{k}} \frac{k^{2}}{\left(k_{n}^{2}+k^{2}\right)^{2}}=\frac{D-1}{2} \int_{\mathbf{k}} \frac{1}{k_{n}^{2}+k^{2}} .
$$

As a consequence the factorized structures on the $3 \mathrm{rd}$ and 5 th rows of eq. (3.23) are proportional to $4-D=2 \epsilon$, and since the sum-integrals $\oiint_{Q} 1 / Q^{2}=T^{2} / 12+\mathcal{O}(\epsilon)$ and $\mathbb{\&}_{\{Q\}} 1 / Q^{2}=$ $-T^{2} / 24+\mathcal{O}(\epsilon)$ are finite in dimensional regularization, there is no contribution from these terms. Defining furthermore

$$
\begin{aligned}
\mathcal{I}_{1}(\tau) & \equiv \mathcal{F}_{K, Q} \frac{e^{i k_{n} \tau}}{Q^{2}(K-Q)^{2}}, \\
\mathcal{I}_{2}(\tau) & \equiv \oiint_{K, Q} \frac{k^{2} e^{i k_{n} \tau}}{K^{2} Q^{2}(K-Q)^{2}}, \\
\mathcal{I}_{3}(\tau) & \equiv \sum_{K, Q} \frac{q_{n}\left(q_{n}-k_{n}\right) e^{i k_{n} \tau}}{K^{2} Q^{2}(K-Q)^{2}}, \\
\mathcal{I}_{4}(\tau) & \equiv \mathcal{F}_{K} \frac{k^{2} e^{i k_{n} \tau}}{K^{2}} \sum_{Q^{\prime}} \frac{e^{i q_{n} \tau}-1}{q_{n}^{2} Q^{2}},
\end{aligned}
$$

and correspondingly $\mathcal{I}_{\{1\}}, \mathcal{I}_{\{2\}}, \mathcal{I}_{\{3\}}$ for the cases that $Q$ is fermionic, and recalling $\mathcal{I}_{5}$ from 
eq. (3.18), we can write the complete (unresummed) contribution as

$$
\begin{aligned}
{\left[\delta G_{\mathrm{E}}^{(4)}(\tau)\right]_{\mathrm{QCD}, \text { naive }} } & =\frac{g_{\mathrm{B}}^{4} C_{F} N_{\mathrm{c}}}{3}\left\{(D-2)\left[-\mathcal{I}_{2}(\tau)-2 \mathcal{I}_{3}(\tau)+\mathcal{I}_{4}(\tau)\right]+\frac{1}{2} \mathcal{I}_{5}(\tau)\right\} \\
& +\frac{g_{\mathrm{B}}^{4} C_{F} N_{\mathrm{f}}}{3}\left[(D-1) \mathcal{I}_{\{1\}}(\tau)-(D-2) \mathcal{I}_{\{2\}}(\tau)+4 \mathcal{I}_{\{3\}}(\tau)\right]
\end{aligned}
$$

\subsection{Matsubara sums, spatial integrals, spectral function}

The next steps are to carry out the Matsubara sums over $k_{n}, q_{n}$ (generalizing eq. (3.5)); to Fourier transform with respect to $\tau$ (eq. (2.5)); to take the discontinuity across the real axis (eq. (2.6) $)$; and to carry out the remaining spatial integrals. We illustrate these steps for one of the structures appearing, $\mathcal{I}_{2}$ of eq. (3.26), in some detail in appendices A.1 A.3. An alternative derivation of the "vacuum part" of the most complicated structure, $\mathcal{I}_{5}$, can be found in appendix A.4. The final results are collected in appendix A.5. Inserting the expressions from eqs. (A.54)-(A.58) of appendix A.5, together with $D=4-2 \epsilon$, we thus get the unresummed bare contribution to the colour-electric spectral function:

$$
\begin{gathered}
{\left[\delta \rho_{\mathrm{E}}^{(4)}(\omega)\right]_{\mathrm{QCD}, \text { naive }}=\frac{g_{\mathrm{B}}^{4} C_{F} N_{\mathrm{c}}}{3}\left\{2(1-\epsilon)\left[-\tilde{\mathcal{I}}_{2}(\omega)-2 \tilde{\mathcal{I}}_{3}(\omega)+\tilde{\mathcal{I}}_{4}(\omega)\right]+\frac{1}{2} \tilde{\mathcal{I}}_{5}(\omega)\right\}} \\
+\frac{g_{\mathrm{B}}^{4} C_{F} N_{\mathrm{f}}}{3}\left[(3-2 \epsilon) \tilde{\mathcal{I}}_{\{1\}}(\omega)-2(1-\epsilon) \tilde{\mathcal{I}}_{\{2\}}(\omega)+4 \tilde{\mathcal{I}}_{\{3\}}(\omega)\right] \\
=\frac{g_{\mathrm{B}}^{4} C_{F} N_{\mathrm{c}}}{3}\left\{\frac{\omega^{3} \mu^{-4 \epsilon}}{(4 \pi)^{3}}\left[\frac{22}{3}\left(\frac{1}{\epsilon}+2 \ln \frac{\bar{\mu}^{2}}{4 \omega^{2}}\right)+\frac{364}{9}-\frac{16 \pi^{2}}{3}\right]\right. \\
+\frac{1}{4 \pi^{3}} \int_{0}^{\infty} \mathrm{d} q n_{\mathrm{B}}(q)\left[\left(q^{2}+2 \omega^{2}\right) \ln \left|\frac{q+w}{q-w}\right|+q \omega\left(\ln \frac{\left|q^{2}-\omega^{2}\right|}{\omega^{2}}-1\right)\right. \\
+\frac{g_{\mathrm{B}}^{4} C_{F} N_{\mathrm{f}}}{3}\left\{\frac{\omega^{3} \mu^{-4 \epsilon}}{(4 \pi)^{3}}\left[-\frac{4}{3}\left(\frac{1}{\epsilon}+2 \ln \frac{\bar{\mu}^{2}}{4 \omega^{2}}\right)-\frac{52}{9}\right]\right. \\
+\frac{1}{4 \pi^{3}} \int_{0}^{\infty} \mathrm{d} q n_{\mathrm{F}}(q)\left[\left(q^{2}+\frac{\omega^{2}}{2}\right) \ln \left|\frac{q+w}{q-w}\right|+q \omega\left(\ln \frac{\left|q^{2}-\omega^{2}\right|}{\omega^{2}}-1\right)\right\}
\end{gathered}
$$

To renormalize this, we need the generalization of eq. (3.9) with effects of $\mathcal{O}(\epsilon)$ included, which can be obtained from eq. (A.21), taking into account the $D-2$ from eq. (3.8):

$$
\left[\rho_{\mathrm{E}}^{(2)}(\omega)\right]_{\mathrm{QCD}, \text { naive }}=\frac{g_{\mathrm{B}}^{2} C_{F}}{6 \pi} \omega^{3} \mu^{-2 \epsilon}\left[1+\epsilon\left(\ln \frac{\bar{\mu}^{2}}{4 \omega^{2}}+1\right)\right] \text {. }
$$

Inserting here the bare gauge coupling from eq. (2.1) and re-expanding in terms of the renormalized gauge coupling, we get an additional contribution which removes the divergences 
from eq. (3.31):

$$
\left[\delta \rho_{\mathrm{E}}^{(4, \mathrm{ct})}(\omega)\right]_{\mathrm{QCD}, \text { naive }}=\frac{g^{4} C_{F}}{3} \frac{\omega^{3} \mu^{-4 \epsilon}}{(4 \pi)^{3}}\left(-\frac{22 N_{\mathrm{c}}}{3}+\frac{4 N_{\mathrm{f}}}{3}\right)\left(\frac{1}{\epsilon}+\ln \frac{\bar{\mu}^{2}}{4 \omega^{2}}+1\right) .
$$

The sum of eqs. (3.31) and (3.33) is our full result for the unresummed next-to-leading order contribution to $\rho_{\mathrm{E}}(\omega)$; it is reproduced in complete form in eq. (4.2) below.

\subsection{Hard Thermal Loop resummation}

The result obtained so far (given explicitly in eq. (4.2) below) contains no infrared divergences, so that in the regime $\omega \gtrsim T$ there is no need for a resummation to be carried out. Nevertheless, we would like to extend the applicability of the result also to the regime $\omega \lesssim g T$ in which collective phenomena like Debye screening play an important role. The reason for this desire is on one hand formal, allowing us to cross-check that the framework is in principle welldefined in this limit as well; and on the other hand practical, permitting us to compare with existing results in the literature. In the regime $\omega \lesssim g T$, logarithmic divergences do appear and a resummation becomes necessary.

The way to consistently carry out the resummation is dictated by eq. (2.9). The practical computations are somewhat cumbersome; given that they also have no bearing on our main result (i.e. the behaviour of the spectral function in the regime $\omega \gtrsim T$ ), we relegate the discussion to appendix B. The main results can be found in eqs. (4.3), (4.6) below, with a notation as introduced in eq. (4.1).

\section{Results}

In this section we collect together the results from the previous section and the appendices, and write down our final formulae for the colour-electric spectral function.

\subsection{Full expression}

In order to write down the full result, we rephrase eq. (2.9) as

$$
\begin{aligned}
\rho_{\mathrm{E}}(\omega) & =\left[\left(\rho_{\mathrm{E}}\right)_{\mathrm{QCD}}-\left(\rho_{\mathrm{E}}\right)_{\mathrm{HTL}}\right]_{\text {naive }}+\left(\rho_{\mathrm{E}}\right)_{\mathrm{HTL}, \text { resum }} \\
& =\left[\left(\rho_{\mathrm{E}}\right)_{\mathrm{QCD}}-\left(\rho_{\mathrm{E}}\right)_{\mathrm{HTL}}\right]_{\text {naive }}+\left(\rho_{\mathrm{E}}\right)_{\mathrm{IR}, \text { resum }}+\left[\left(\rho_{\mathrm{E}}\right)_{\mathrm{HTL}}-\left(\rho_{\mathrm{E}}\right)_{\mathrm{IR}}\right]_{\mathrm{resum}} \\
& =\underbrace{\left(\rho_{\mathrm{E}}\right)_{\mathrm{QCD} \text {, naive }}}_{\text {eq. (4.2) }}+\underbrace{\left(\rho_{\mathrm{E}}\right)_{\mathrm{IR} \text {, resum }}-\left(\rho_{\mathrm{E}}\right)_{\mathrm{HTL}, \text { naive }}}_{\text {eq. (4.3) }}+\underbrace{\left(\rho_{\mathrm{E}}\right)_{\mathrm{HTL}, \text { resum }}-\left(\rho_{\mathrm{E}}\right)_{\mathrm{IR}, \text { resum }}}_{\text {eq. (4.6) }},
\end{aligned}
$$

where we left out the arguments $\omega$ for brevity. The term $\left(\rho_{\mathrm{E}}\right)_{\mathrm{IR} \text {, resum }}$, added and subtracted in eq. (4.1), is defined to be the HTL-resummed spectral function but computed only up to 
the leading (linear) order in a Taylor expansion around $\omega=0$. The rationale for this split-up is that all three structures are ultraviolet and infrared finite (i.e. contain no $1 / \epsilon$ divergences) and that the last term, eq. (4.6), is readily available from the literature (cf. below).

The first term of eq. (4.1) is the sum of eqs. (3.9), (3.31), (3.33):

$$
\begin{aligned}
{\left[\rho_{\mathrm{E}}(\omega)\right]_{\mathrm{QCD}, \text { naive }}=} & \frac{g^{2} C_{F} \omega^{3}}{6 \pi}\left\{1+\frac{g^{2}}{(4 \pi)^{2}}\left[-N_{\mathrm{f}}\left(\frac{2}{3} \ln \frac{\bar{\mu}^{2}}{4 \omega^{2}}+\frac{20}{9}\right)\right.\right. \\
& \left.\left.+N_{\mathrm{c}}\left(\frac{11}{3} \ln \frac{\bar{\mu}^{2}}{4 \omega^{2}}+\frac{149}{9}-\frac{8 \pi^{2}}{3}\right)\right]\right\} \\
& +\frac{g^{2} C_{F}}{6 \pi} \frac{g^{2}}{2 \pi^{2}}\left\{\quad\left[\left(q^{2}+\frac{\omega^{2}}{2}\right) \ln \left|\frac{q+w}{q-w}\right|+q \omega\left(\ln \frac{\left|q^{2}-\omega^{2}\right|}{\omega^{2}}-1\right)\right]\right. \\
& +N_{\mathrm{f}} \int_{0}^{\infty} \mathrm{d} q n_{\mathrm{F}}(q)\left[q^{2}+2 \omega^{2}\right) \ln \left|\frac{q+w}{q-w}\right|+q \omega\left(\ln \frac{\left|q^{2}-\omega^{2}\right|}{\omega^{2}}-1\right) \\
& +N_{\mathrm{c}} \int_{0}^{\infty} \mathrm{d} q n_{\mathrm{B}}(q) \\
& \left.\left.+\frac{\omega^{4}}{q} \mathbb{P}\left(\frac{1}{q+\omega} \ln \frac{q+\omega}{\omega}+\frac{1}{q-\omega} \ln \frac{\omega}{|q-\omega|}\right)\right]\right\} .
\end{aligned}
$$

We stress that this spectral function contains no "transport peak" $\sim \omega \delta(\omega)$ (in fact none of the master sum-integrals appearing in our computation have such terms). In addition, the function $\tilde{G}_{\mathrm{E}}\left(\omega_{n}\right)$, the cut of which determines $\rho_{\mathrm{E}}(\omega)$, contains no term constant in $\omega_{n}$, which would correspond to a contact term in the Euclidean correlator $G_{\mathrm{E}}(\tau)$. Therefore $\rho_{\mathrm{E}}(\omega)$ contains all the information characterizing the correlator in an "unproblematic" form (see ref. [18] for a recent discussion of this issue in a context where complications do appear).

The second term of eq. (4.1) is a combination of eqs. (B.6), (B.14), (B.20):

$$
\left[\rho_{\mathrm{E}}(\omega)\right]_{\mathrm{IR}, \text { resum }}-\left[\rho_{\mathrm{E}}(\omega)\right]_{\mathrm{HTL}, \text { naive }}=\frac{g^{2} C_{F}}{6 \pi}\left[-\omega^{3}+\frac{1}{2} \omega m_{\mathrm{E}}^{2}\left(\ln \frac{2 \omega}{m_{\mathrm{E}}}-1\right)\right],
$$

where $m_{\mathrm{E}}$ is the Debye mass parameter,

$$
m_{\mathrm{E}}^{2}=\left(\frac{N_{\mathrm{f}}}{6}+\frac{N_{\mathrm{c}}}{3}\right) g^{2} T^{2} .
$$

The term of $\mathcal{O}\left(g^{2}\right)$ cancels in the sum of eqs. (4.2), (4.3); this term is contained in a modified form within eq. (4.6) (cf. eq. (4.7)). It is also interesting to note that, inserting $m_{\mathrm{E}}^{2}$ as expressed in eq. (B.5), the next-to-leading order contribution in eq. (4.3) can be rewritten as

$$
\left[\rho_{\mathrm{E}}^{(4)}(\omega)\right]_{\mathrm{IR}, \text { resum }}-\left[\rho_{\mathrm{E}}^{(4)}(\omega)\right]_{\mathrm{HTL}, \text { naive }}=\frac{g^{2} C_{F}}{6 \pi} \frac{g^{2}}{2 \pi^{2}} \int_{0}^{\infty} \mathrm{d} q\left[N_{\mathrm{f}} n_{\mathrm{F}}(q)+N_{\mathrm{c}} n_{\mathrm{B}}(q)\right] q \omega\left(\ln \frac{4 \omega^{2}}{m_{\mathrm{E}}^{2}}-2\right),
$$

which nicely combines with some of the terms in eq. (4.2). 
The remaining ingredient, the HTL-resummed contribution beyond the linear term in $\omega$, i.e. $\left(\rho_{\mathrm{E}}\right)_{\mathrm{HTL}}$, resum $-\left(\rho_{\mathrm{E}}\right)_{\mathrm{IR} \text {, resum }}$, was determined in ref. [4]. With a minor change of notation, eq. (3.8) of ref. [4] can be expressed as

$$
\begin{aligned}
& {\left[\rho_{\mathrm{E}}^{(4)}(\omega)\right]_{\mathrm{HTL}, \text { resum }}-\left[\rho_{\mathrm{E}}^{(4)}(\omega)\right]_{\mathrm{IR}, \text { resum }}=\frac{g^{2} C_{F} m_{\mathrm{E}}^{2} \omega}{6 \pi} \times\{} \\
& \quad \int_{\hat{\omega}}^{\infty} \frac{\mathrm{d} \hat{k} \hat{k}}{2} \frac{\hat{\omega}^{2}\left(1-\frac{\hat{\omega}^{2}}{\hat{k}^{2}}\right)}{\left(\hat{k}^{2}-\hat{\omega}^{2}+\frac{1}{2}\left[\frac{\hat{\omega}^{2}}{\hat{k}^{2}}+\frac{\hat{\omega}}{2 \hat{k}}\left(1-\frac{\hat{\omega}^{2}}{\hat{k}^{2}}\right) \ln \frac{\hat{k}+\hat{\omega}}{\hat{k}-\hat{\omega}}\right]\right)^{2}+\left(\frac{\hat{\omega} \pi}{4 \hat{k}}\right)^{2}\left(1-\frac{\hat{\omega}^{2}}{\hat{k}^{2}}\right)^{2}} \\
& \quad+\int_{0}^{\infty} \frac{\mathrm{d} \hat{k} \hat{k}^{3}}{2}\left[\frac{1(\hat{k}-\hat{\omega})}{\left(\hat{k}^{2}+1-\frac{\hat{\omega}}{2 \hat{k}} \ln \frac{\hat{k}+\hat{\omega}}{\hat{k}-\hat{\omega})^{2}+\left(\frac{\hat{\omega} \pi}{2 \hat{k}}\right)^{2}}-\frac{1}{\left(\hat{k}^{2}+1\right)^{2}}\right]}\right. \\
& \left.+\left.\frac{2 \hat{\omega} \hat{k}_{T}^{3}\left(\hat{\omega}^{2}-\hat{k}_{T}^{2}\right)}{\left|3\left(\hat{k}_{T}^{2}-\hat{\omega}^{2}\right)^{2}-\hat{\omega}^{2}\right|}\right|_{\hat{k}_{T}^{2}-\hat{\omega}^{2}+\frac{1}{2}\left[\frac{\hat{\omega}^{2}}{\hat{k}_{T}^{2}}+\frac{\hat{\omega}}{2 \hat{k}_{T}}\left(1-\frac{\hat{\omega}^{2}}{\hat{k}_{T}^{2}}\right) \ln \frac{\hat{\omega}+\hat{k}_{T}}{\hat{\omega}-\hat{k}_{T}}\right]=0}\right\} \\
& \left.+\left.\frac{\hat{k}_{E}^{3}\left(\hat{\omega}^{2}-\hat{k}_{E}^{2}\right)}{\hat{\hat{\omega}}\left|3\left(\hat{k}_{E}^{2}-\hat{\omega}^{2}\right)+1\right|}\right|_{\hat{k}_{E}^{2}+1-\frac{\hat{\omega}}{2 \hat{k}_{E}} \ln \frac{\hat{\omega}+\hat{k}_{E}}{\hat{\omega}-\hat{k}_{E}}=0}\right\}
\end{aligned}
$$

where $\hat{\omega} \equiv \omega / m_{\mathrm{E}}$ and $\hat{k} \equiv k / m_{\mathrm{E}}$, and the expression within the curly brackets is dimensionless.

For $\hat{\omega} \gg 1$ it can be checked, perhaps most easily numerically, that the expression within the curly brackets behaves as

$$
\{\ldots\} \stackrel{\hat{\omega} \gg 1}{=} \hat{\omega}^{2}+\frac{1}{2}\left(\ln \frac{1}{2 \hat{\omega}}+1\right)+\mathcal{O}\left(\frac{1}{\hat{\omega}^{2}}\right) .
$$

This cancels against eq. (4.3), so that for $\omega \gg m_{\mathrm{E}}$ only the naive QCD contribution of eq. (4.2) is left over, as must be the case.

\subsection{Ultraviolet asymptotics}

An important application of the general result of sec. 4.1 is that it determines the asymptotic $\left(\omega \gg\left\{T, m_{\mathrm{E}}, \Lambda_{\overline{\mathrm{MS}}}\right\}\right.$ ) behaviour of the colour-electric spectral function. In fact, as explained in connection with eq. (4.7), for large frequencies $\omega \gg m_{\mathrm{E}}$ we can make directly use of the unresummed expression, eq. (4.2). Expanding the integrand of eq. (4.2) in $q / \omega$ and making use of

$$
\int_{0}^{\infty} \frac{\mathrm{d} x x^{3}}{e^{x}-1}=\frac{\pi^{4}}{15}, \quad \int_{0}^{\infty} \frac{\mathrm{d} x x^{3}}{e^{x}+1}=\frac{7 \pi^{4}}{120}
$$

it can be seen that the thermal part of the spectral function disappears at large frequencies:

$$
\rho_{\mathrm{QCD}}(\omega) \stackrel{\omega \gg T}{=}\left[\rho_{\mathrm{QCD}}(\omega)\right]_{T=0}+\frac{g^{4} C_{F}}{6 \pi} \frac{\pi^{2} T^{4}}{180 \omega}\left(7 N_{\mathrm{f}}-11 N_{\mathrm{c}}\right) .
$$


Such a power-law decay of the thermal correction at $\omega \gg T$ is in accordance with the general results of ref. [19], although it is not clear to us whether a precise relation to the Operator Product Expansion as used in ref. [19] can be established for our non-local correlator. The term denoted by " $T=0$ " in eq. (4.9), on the other hand, is temperature independent, and given by the first two rows of eq. (4.2):

$$
\begin{aligned}
{\left[\rho_{\mathrm{QCD}}(\omega)\right]_{T=0}=\frac{g^{2} C_{F} \omega^{3}}{6 \pi}\left\{1+\frac{g^{2}}{(4 \pi)^{2}}\right.} & {\left[-N_{\mathrm{f}}\left(\frac{2}{3} \ln \frac{\bar{\mu}^{2}}{4 \omega^{2}}+\frac{20}{9}\right)\right.} \\
+ & \left.\left.N_{\mathrm{c}}\left(\frac{11}{3} \ln \frac{\bar{\mu}^{2}}{4 \omega^{2}}+\frac{149}{9}-\frac{8 \pi^{2}}{3}\right)\right]\right\}+\mathcal{O}\left(g^{6}\right) .
\end{aligned}
$$

A rough estimate can be obtained by setting $\bar{\mu} \approx 2 \omega$ and inserting the 1-loop running coupling,

$$
\left[\frac{\rho_{\mathrm{QCD}}(\omega)}{\omega^{3}}\right]_{T=0} \simeq \frac{4 \pi C_{F}}{\left(11 N_{\mathrm{c}}-2 N_{\mathrm{f}}\right) \ln \left(2 \omega / \Lambda_{\overline{\mathrm{MS}}}\right)}\left\{1-\frac{\left(24 \pi^{2}-149\right) N_{\mathrm{c}}+20 N_{\mathrm{f}}}{6\left(11 N_{\mathrm{c}}-2 N_{\mathrm{f}}\right) \ln \left(2 \omega / \Lambda_{\overline{\mathrm{MS}}}\right)}\right\} .
$$

Numerical evaluations are discussed in sec. 4.4,

It is perhaps appropriate to stress that the first omitted term in eq. (4.7) (which is not explicitly cancelled within our computation) leads to a contribution of the type $\delta \rho_{\mathrm{E}} \sim g^{2} m_{\mathrm{E}}^{4} / \omega$, which is parametrically of higher order than the $T$-dependent part of eq. (4.9). Hence, at $\mathcal{O}\left(\alpha_{s}^{2}\right)$, ultraviolet asymptotics is indeed completely determined by hard modes. This is again in accordance with the general analysis of ref. [19]

\subsection{Infrared asymptotics}

We next discuss the infrared $\left(\omega \ll m_{\mathrm{E}}\right.$ ) behaviour of the spectral function, in the range $T \gg \Lambda_{\overline{\mathrm{MS}}}$. Although we have nothing to add to the existing results in this regime [7, 8], it is comforting to crosscheck that we can at least reproduce the expression at $\mathcal{O}\left(\alpha_{s}^{2}\right)$. Since the computation of ref. [7] was carried out in Coulomb gauge whereas we have described a covariant gauge computation, this also serves as a further cross-check of gauge independence.

In the regime $\omega \ll m_{\mathrm{E}}$ the " $T=0$ " contribution (eq. (4.10) ) is subdominant compared with the thermal one, which is of $\mathcal{O}\left(g^{2} m_{\mathrm{E}}^{2} \omega\right)$. Expanding the thermal integrand of eq. (4.2) in $\omega / q$ and making use of

$$
\begin{array}{ll}
\int_{0}^{\infty} \frac{\mathrm{d} x x}{e^{x}-1}=\frac{\pi^{2}}{6}, & \int_{0}^{\infty} \frac{\mathrm{d} x x \ln x}{e^{x}-1}=\frac{\pi^{2}}{6}\left[1-\gamma_{\mathrm{E}}+\frac{\zeta^{\prime}(2)}{\zeta(2)}\right] \\
\int_{0}^{\infty} \frac{\mathrm{d} x x}{e^{x}+1}=\frac{\pi^{2}}{12}, & \int_{0}^{\infty} \frac{\mathrm{d} x x \ln x}{e^{x}+1}=\frac{\pi^{2}}{12}\left[\ln 2+1-\gamma_{\mathrm{E}}+\frac{\zeta^{\prime}(2)}{\zeta(2)}\right]
\end{array}
$$

we obtain the infrared behaviour of the unresummed QCD result, expressed now in terms of 
the momentum diffusion coefficient of eq. (2.7):

$$
\begin{aligned}
& {[\kappa]_{\mathrm{QCD}, \text { naive }}=\lim _{\omega \rightarrow 0}\left[\frac{2 T \rho_{\mathrm{E}}(\omega)}{\omega}\right]_{\mathrm{QCD}, \text { naive }}} \\
& \quad \approx \frac{g^{4} C_{F} T^{3}}{6 \pi}\left\{\frac{N_{\mathrm{c}}}{3}\left[\ln \frac{T}{\omega}+\frac{3}{2}-\gamma_{\mathrm{E}}+\frac{\zeta^{\prime}(2)}{\zeta(2)}\right]+\frac{N_{\mathrm{f}}}{6}\left[\ln \frac{2 T}{\omega}+\frac{3}{2}-\gamma_{\mathrm{E}}+\frac{\zeta^{\prime}(2)}{\zeta(2)}\right]\right\} .
\end{aligned}
$$

The symbol " $\approx$ " signals that the logarithmically divergent term has been kept finite on the right-hand side. Similarly, from eq. (4.3), the HTL contribution to $\kappa$ is

$$
[\kappa]_{\mathrm{HTL}, \text { resum }}-[\kappa]_{\mathrm{HTL}, \text { naive }} \approx \frac{g^{2} C_{F} T}{6 \pi} m_{\mathrm{E}}^{2}\left[\ln \frac{2 \omega}{m_{\mathrm{E}}}-1\right] .
$$

Summing together eqs. (4.14) and (4.15), we reproduce the result of ref. [7]:

$$
\kappa=\frac{g^{2} C_{F} T}{6 \pi} m_{\mathrm{E}}^{2}\left(\ln \frac{2 T}{m_{\mathrm{E}}}+\frac{1}{2}-\gamma_{\mathrm{E}}+\frac{\zeta^{\prime}(2)}{\zeta(2)}+\frac{N_{\mathrm{f}} \ln 2}{2 N_{\mathrm{c}}+N_{\mathrm{f}}}\right)(1+\mathcal{O}(g)) .
$$

As indicated, corrections to this expression start already at $\mathcal{O}(g)$, and have been determined in ref. [8, but in the infrared regime these go beyond the accuracy of our computation.

We note that if $g$ is not small $\left(T / m_{\mathrm{E}}\right.$ is not large), the expression in eq. (4.16) can become negative. This unphysical behaviour is a result of extrapolating the asymptotic weak-coupling expression beyond its range of applicability; for $N_{\mathrm{c}}=3, N_{\mathrm{f}}=0$, the problem occurs already at $g \gtrsim 1.05$ and is another reflection of the exceptionally poor convergence of the weak-coupling expansion of $\kappa$ (see also ref. [8]). Given that the unresummed expression (eq. (4.14)) does stay positive and in fact diverges as $\omega \rightarrow 0$, the HTL-resummation (eq. (4.15)) in some sense "over-corrects" the result in this regime.

\subsection{Numerical evaluation}

In order to finally evaluate our result (eq. (4.2) $+(4.3)+(4.6)$, cf. eq. (4.1) ) numerically, we need to insert some value for the running coupling $g^{2}$. It is only in the regime $\omega \gg T$ that two subsequent orders with the same functional form are at our disposal, so that some kind of a scale optimization is possible; we can then define $\bar{\mu}_{\text {opt }}$ for $g^{2}$ from the "fastest apparent convergence" or "principal of minimal sensitivity" criterion based on eq. (4.10):

$$
\ln \left(\bar{\mu}_{\mathrm{opt}(\omega)}\right) \equiv \ln (2 \omega)+\frac{\left(24 \pi^{2}-149\right) N_{\mathrm{c}}+20 N_{\mathrm{f}}}{6\left(11 N_{\mathrm{c}}-2 N_{\mathrm{f}}\right)}
$$

a structure indirectly also visible in eq. (4.11). In the infrared regime $\omega \ll T$ such an exercise is not possible but we nevertheless need a value for $g^{2}$; we choose it from a context in which a next-to-leading order computation does exist, namely that of "EQCD" (cf. ref. [20] and 

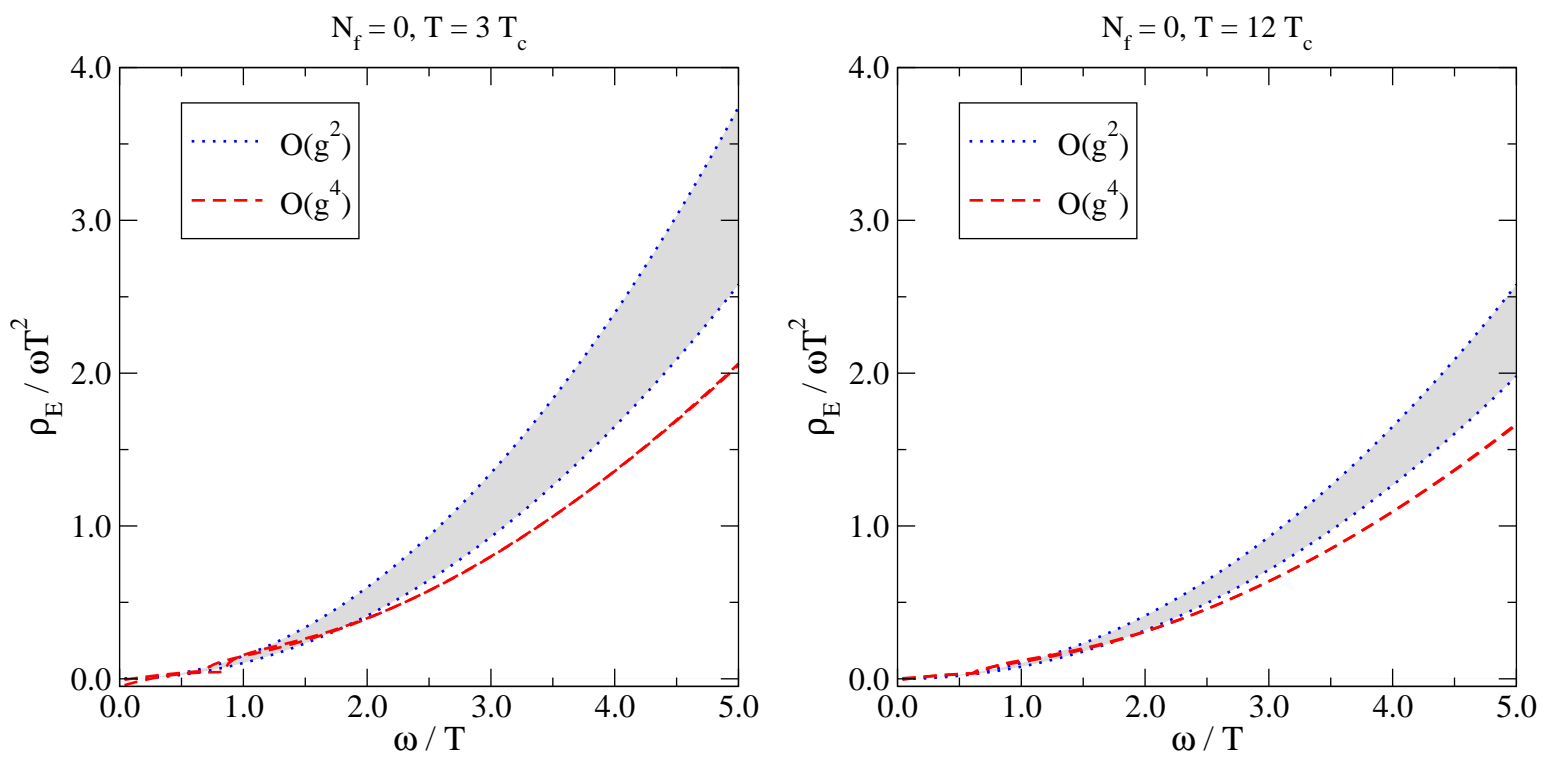

Figure 2: A numerical evaluation of $\rho_{\mathrm{E}}(\omega)$, eq. (4.1), in units of $\omega T^{2}$, for $N_{\mathrm{c}}=3, N_{\mathrm{f}}=0, T=$ $3.75 \Lambda_{\overline{\mathrm{MS}}}$ (left) and $T=15 \Lambda_{\overline{\mathrm{MS}}}$ (right), corresponding to $T \approx 3 T_{\mathrm{c}}$ and $T \approx 12 T_{\mathrm{c}}$, respectively. The gauge coupling has been fixed as explained around eqs. (4.17), (4.18), and the error bar reflects the corresponding uncertainty. (In the $\mathcal{O}\left(g^{2}\right)$ result the "optimal" scale is fixed to the thermal value of eq. (4.18), i.e. does not change with the frequency.) A slightly negative intercept at $\omega \rightarrow 0$ is an artifact of the truncated weak-coupling expansion, cf. discussion below eq. (4.16).

references therein):2

$$
\ln \left(\bar{\mu}_{\mathrm{opt}(T)}\right) \equiv \ln (4 \pi T)-\gamma_{\mathrm{E}}-\frac{N_{\mathrm{c}}-8 \ln 2 N_{\mathrm{f}}}{2\left(11 N_{\mathrm{c}}-2 N_{\mathrm{f}}\right)} .
$$

Comparing eqs. (4.17) and (4.18) we see that we can switch from one regime to another at $\omega \sim T$, for instance at $\omega \approx 0.8903 T$ for $N_{\mathrm{f}}=0$. In order to get an error band for the uncertainty related to the choice of $g^{2}$, we use the 2-loop $g^{2}$, with the scale parameter varied in the range $(0.5 \ldots 2.0) \times \bar{\mu}_{\text {opt }}$. Since the Debye mass parameter plays no role in the range $\omega \gg T$ that we are mostly interested in, we fix it in the simplest way imaginable, through eq. (4.4) with the gauge coupling evaluated as described above.

The outcome of a numerical evaluation of eq. (4.1) is plotted in fig. 2, in units of $\omega T^{2}$. We observe that because of the running of the gauge coupling the next-to-leading order result falls below the leading-order one in the ultraviolet domain, $\omega \gg T$. The dependence on the scale choice is also drastically reduced, becoming (perhaps rather surprisingly) practically invisible as soon as $\omega \gtrsim T$.

In the infrared domain, $\omega \ll T$, the next-to-leading order correction eventually overtakes the leading-order result, and even the HTL-resummed expression shows very slow convergence

\footnotetext{
${ }^{2}$ For EQCD even a NNLO computation exists 21] but that goes beyond the accuracy of the present study.
} 

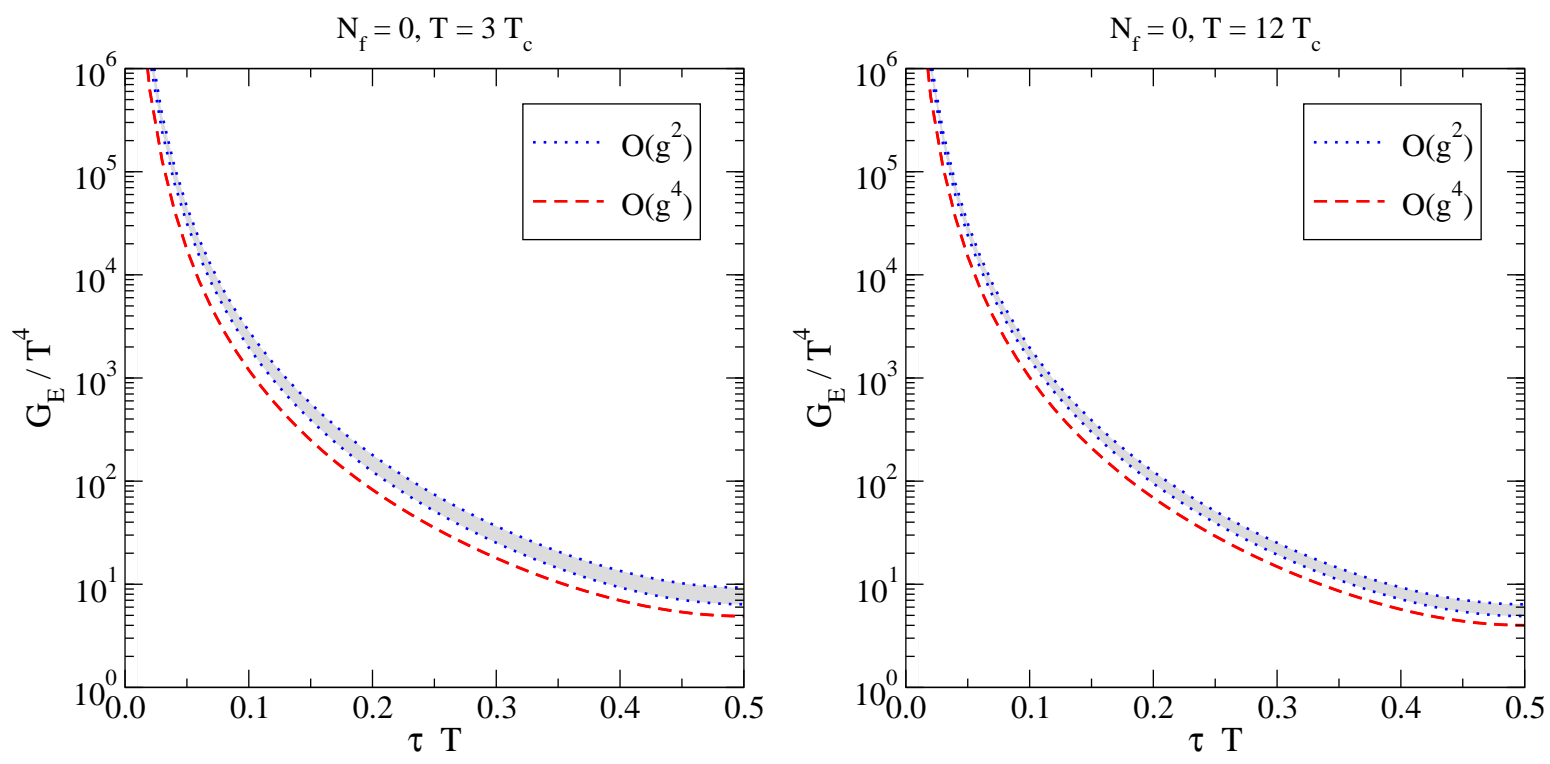

Figure 3: A numerical evaluation of $G_{\mathrm{E}}(\tau)$, in units of $T^{4}$, for $N_{\mathrm{c}}=3, N_{\mathrm{f}}=0, T=3.75 \Lambda_{\overline{\mathrm{MS}}}$ (left) and $T=15 \Lambda_{\overline{\mathrm{MS}}}$ (right), corresponding to $T \approx 3 T_{\mathrm{c}}$ and $T \approx 12 T_{\mathrm{c}}$, respectively. The gauge coupling has been fixed as explained around eqs. (4.17), (4.18), and the error bar reflects the corresponding uncertainty. (In the $\mathcal{O}\left(g^{2}\right)$ result the "optimal" scale is fixed to the thermal value of eq. (4.18), i.e. does not change with the frequency.) On the logarithmic scale used a clear temperature-dependence is visible only around the middle of the interval, $\tau T \approx 0.5$.

(cf. ref. [8]). Nevertheless, fig. 2 does illustrate an important point: even at weak coupling there is no transport peak around the origin; rather $\rho_{\mathrm{E}}(\omega) / \omega$ displays a relatively flat behavior at $\omega \lesssim T$. More precise studies of the infrared domain have so far been carried out within classical lattice gauge theory [11] as well as within $\mathcal{N}=4$ Super-Yang-Mills theory at infinite 't Hooft coupling [3, 10].

In fig. 3 we plot the Euclidean correlators $G_{\mathrm{E}}(\tau)$ corresponding to the spectral function $\rho_{\mathrm{E}}(\omega)$ of fig. 2, obtained through eq. (2.8). The leading-order result can be given in a closed form [4,

$$
G_{\mathrm{E}}^{(2)}(\tau)=g^{2} C_{F} \pi^{2} T^{4}\left[\frac{\cos ^{2}(\pi \tau T)}{\sin ^{4}(\pi \tau T)}+\frac{1}{3 \sin ^{2}(\pi \tau T)}\right]
$$

whereas the next-to-leading order correction $G_{\mathrm{E}}^{(4)}$ has been evaluated numerically. The $\omega$ dependent difference of $\rho_{\mathrm{E}}$ at $\mathcal{O}\left(g^{2}\right)$ and $\mathcal{O}\left(g^{4}\right)$ in fig. 2 has converted to a practically $\tau$ independent shift on the logarithmic scale of fig. 3.

We note that, parametrically, the range $\omega \lesssim m_{\mathrm{E}} \ll 2 T$ gives a contribution to eq. (2.8) of magnitude

$$
\left[\delta G_{\mathrm{E}}^{(4)}(\tau)\right]_{\mathrm{HTL}} \sim \int_{0}^{m_{\mathrm{E}}} \frac{\mathrm{d} \omega}{\pi} \frac{T \rho_{\mathrm{E}}(\omega)}{\omega} \sim g^{2} m_{\mathrm{E}}^{3} T \sim \mathcal{O}\left(g^{5} T^{4}\right)
$$


cf. eq. (4.15), which is formally of higher order than the ultraviolet contribution, $\mathcal{O}\left(g^{4} T^{4}\right)$. This is also reflected through the fact the infrared divergence of eq. (4.14) is integrable at small $\omega$. Nevertheless, the next ultraviolet contributions are of higher order still, $\mathcal{O}\left(g^{6} T^{4}\right)$, so we can already meaningfully include even the infrared part of $\rho_{\mathrm{E}}(\omega)$ in our numerical evaluation of $G_{\mathrm{E}}$. In practice, of course, the scale $m_{\mathrm{E}}$ is not smaller than $\sim 2 T$ at phenomenologically interesting temperatures, and the infrared contribution should not be substantially suppressed.

\section{Colour-electric correlator in the strong-coupling expansion}

In the confined (low-temperature) phase of pure $\mathrm{SU}\left(N_{\mathrm{c}}\right)$ gauge theory, the Euclidean correlator of eq. (2.4) looks singular, because the expectation value in the denominator vanishes. We wish to demonstrate that despite this appearance the ratio could actually remain finite, if defined through a suitable limiting procedure by explicitly breaking the $\mathrm{Z}\left(N_{\mathrm{c}}\right)$ center symmetry (an obvious possibility would be the addition of dynamical quarks). As a tool we use the lattice strong-coupling expansion, which converges (deep enough) in the confined phase. The result also yields an interesting functional dependence on $\tau$, which can be contrasted with the weak-coupling result of fig. 3 ,

Defining the strong-coupling expansion requires the use of lattice regularization, and hence also a discretization of the correlator of eq. (2.4). The discretization is obviously not unique; we choose the possibility proposed in eq. (4.2) of ref. [4, viz.

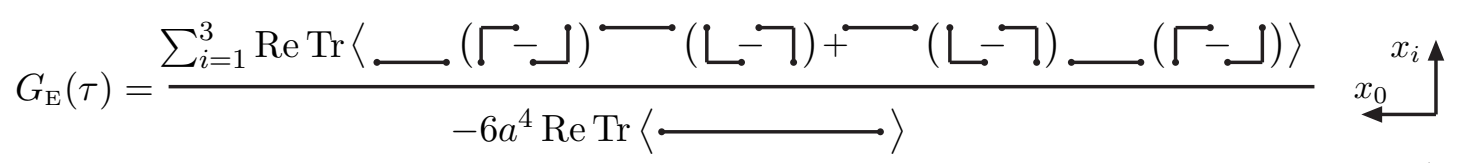

where $a$ is the lattice spacing and we have used a graphical notation with lines indicating parallel transporters (for more details see ref. [4]). Let us also define the auxiliary correlator

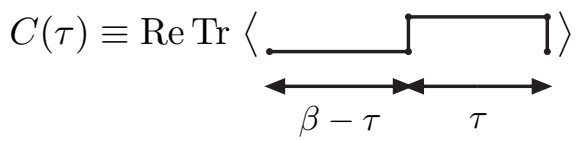

in which the vertical lines have the length of one lattice spacing. If $C(0) \neq 0$, we can then write

$$
G_{\mathrm{E}}(\tau)=\frac{C(\tau-a)+C(\tau+a)-2 C(\tau)}{a^{4} C(0)} .
$$

Note that this expression makes sense only for $\tau \geq a$.

In order to guarantee that $C(0)$ is non-zero, we add a small "source term" to the (Wilson) action, thus breaking the $\mathrm{Z}\left(N_{\mathrm{c}}\right)$ symmetry explicitly. We believe that the precise from of this regulator does not matter, and in practice we choose the simplest possibility, adding to 
the action just the volume sum of the Polyakov loop itself, multiplied by a small "magnetic field", to be denoted by $\kappa$. Then (if $N_{\tau} \geq 4$ ),

$$
C(\tau)=\kappa\left[u^{\frac{\tau}{a}}+u^{\frac{\beta-\tau}{a}}\right]\left(1+\mathcal{O}\left(u^{4}\right)\right)+\mathcal{O}\left(\kappa^{2}\right)
$$

where $N_{\tau} \equiv \beta / a$ and $u$ is a variable naturally arising in the character expansion (for details see, e.g., ref. [22]),

$$
u \equiv \frac{1}{N_{\mathrm{c}}} \operatorname{Tr}\left\langle\left\langle P_{i j}\right\rangle\right\rangle
$$

Here $P_{i j}$ denotes an elementary plaquette in the $(i, j)$-plane, and the expectation value is defined as $\left\langle\left\langle P_{i j}\right\rangle\right\rangle \equiv \int \mathrm{d} P_{i j} P_{i j} \exp \left(\frac{\beta_{\mathrm{G}}}{2 N_{\mathrm{c}}} \operatorname{Tr}\left[P_{i j}+P_{i j}^{\dagger}\right]\right) / \int \mathrm{d} P_{i j} \exp \left(\frac{\beta_{\mathrm{G}}}{2 N_{\mathrm{c}}} \operatorname{Tr}\left[P_{i j}+P_{i j}^{\dagger}\right]\right)$. The strongcoupling expansion is an expansion in a small Wilson parameter $\beta_{\mathrm{G}}$, which means that the variable $u$ should also be small: for $N_{\mathrm{c}} \geq 3$,

$$
u=\frac{\beta_{\mathrm{G}}}{2 N_{\mathrm{c}}^{2}}+\mathcal{O}\left(\beta_{\mathrm{G}}^{2}\right)
$$

Incidentally, we have also worked out the correction of $\mathcal{O}\left(u^{4}\right)$ to eq. (5.4); it amounts to the replacement $u^{x} \rightarrow\left(1+4 x u^{4}\right) u^{x}$, with $x=\frac{\tau}{a}$ and $x=\frac{\beta-\tau}{a}$, but this does not change the qualitative behaviour so we do not elaborate on further details here.

Inserting eq. (5.4) into eq. (5.3), we note that the magnetic field $\kappa$ drops out, and a finite result is left over. Moreover, inserting some small value for $u(0<u \ll 1)$, we obtain a function which is not unlike those in fig. 3, examples are shown in fig. 4. The main differences are that at very low temperatures (very small $u$ ), the exponential suppression around the middle of the interval is much stronger than in the deconfined phase; and that there is little curvature in the plots (i.e. only a single exponential). Taking a discrete Fourier transform and subsequently naively applying eq. (2.6) to it, leads to a spectral function with $\delta$-function peaks at $\omega= \pm \frac{1}{a} \ln u$ but no other structure. This behaviour is completely different from those in fig. 2, the difference demonstrates once again that a high resolution is needed in the determination of $G_{\mathrm{E}}(\tau)$, in order for us to be able to extract the correct non-trivial features of the corresponding spectral function.

In a practical lattice measurement, the numerator and the denominator of eq. (5.3) are evaluated separately. If the breaking of the $\mathrm{Z}\left(N_{\mathrm{c}}\right)$ symmetry is only explicit (not spontaneous) and small, the signal is likely to be very noisy. This is not really a problem from the phenomenological point of view, though, since heavy quark diffusion appears anyways to be a physically meaningful concept only in the deconfined phase where $\mathrm{Z}\left(N_{\mathrm{c}}\right)$ is indeed spontaneously broken (at any non-zero lattice spacing). 


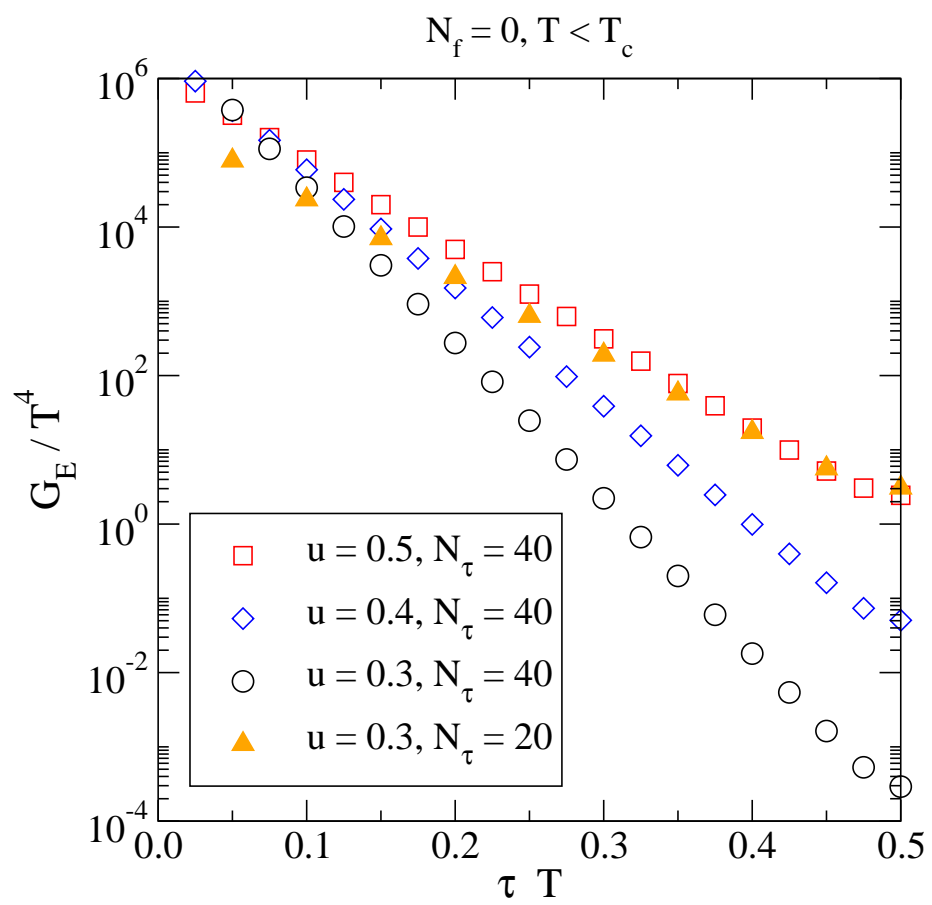

Figure 4: A numerical evaluation of the strong-coupling result for $G_{\mathrm{E}}(\tau)$, eqs. (5.3), (5.4), in units of $T^{4}$. For a fixed $N_{\tau}$, smaller values of $u$ correspond to lower temperatures. The qualitative behaviour can be compared with that in fig. 3. the exponential suppression around the middle of the interval becomes very strong at low temperatures (note that the scales are different in the two figures).

\section{Conclusions and outlook}

The "transport coefficient" related to the correlator of two colour-electric fields along a Polyakov loop determines the momentum diffusion coefficient, $\kappa$, of a heavy quark near rest with respect to a thermal medium. Among all the QCD transport coefficients this is probably the most accessible one from the theoretical point of view; indeed it is the first for which a next-to-leading order weak-coupling expression was obtained [8]. Alas, as the weak-coupling expression itself [8] as well as numerical studies within classical lattice gauge theory [11] show, the weak-coupling expansion converges very slowly at any temperature relevant for heavy ion collision experiments, so a non-perturbative determination would be highly desirable.

On the point of the non-perturbative determination, there is the salient feature that no transport peak exists in the colour-electric spectral function from which $\kappa$ is extracted, $\rho_{\mathrm{E}}(\omega)$, even at weak coupling where spectral functions are in general more singular than in a strongly interacting system. The reason is that there is no (approximately) conserved charge related to the colour-electric field strength. Employing this theoretical insight as a constraint might help to reduce the systematic uncertainties that are inherent to the practical recipes used for 
the analytic continuation from the measured Euclidean correlator to the desired $\rho_{\mathrm{E}}(\omega)$.

On the other hand, the non-perturbative study is faced with the challenge that the absolute magnitude of the correlator is needed for the determination of $\kappa$, which is obtained from the $\omega \rightarrow 0$ intercept of $2 T \rho_{\mathrm{E}}(\omega) / \omega$. This means that the renormalization of the colour-electric field entering the correlator plays an important role.

In principle, the colour-electric field can be renormalized independently of the particular observable considered (perhaps in analogy with procedure for colour-magnetic fields in ref. 23]). However, its discretization is not unique, so it may be useful to have a way to "crosscheck" the normalization of the result directly with the data at hand. For this purpose our asymptotic large- $\omega$ behaviour might turn out to be useful, since the weak-coupling result is relatively accurate at $\omega \gg T$ (at least within $\sim 10 \%$, which would at the current stage be perfectly sufficient as far as $\kappa$ is concerned). Our formula is strictly applicable only in the continuum limit; nevertheless, a practical matching could already be attempted at some intermediate frequency $\omega$ and non-zero lattice spacing $a$, provided that the inequalities $T \ll \omega \ll 1 / a$ can be satisfied. Of course, all of this assumes that some $\rho_{\mathrm{E}}(\omega)$ can be approximately reconstructed from a measured Euclidean correlator $G_{\mathrm{E}}(\tau)$ [16].

Apart from the spectral function (fig. 2), we have also estimated the corresponding Euclidean correlator (figs. 3, 40) which can be directly compared with lattice simulations. The result appears to be most sensitive to thermal effects around the middle of the Euclidean time interval, where its absolute value is very small, posing a challenge for numerical simulations. Nevertheless, with refined techniques a reasonable signal should ultimately be obtainable.

To summarize, it is our (perhaps optimistic) hope that by combining our analytic work with forthcoming numerical studies a qualitative and even approximate quantitative understanding concerning the shape of the spectral function $\rho_{\mathrm{E}}(\omega)$ and in particular the intercept $\kappa=$ $\lim _{\omega \rightarrow 0} 2 T \rho_{\mathrm{E}}(\omega) / \omega$ can eventually be developed.

\section{Acknowledgements}

We are grateful to the BMBF for financial support under the project Heavy Quarks as a Bridge between Heavy Ion Collisions and QCD. M.L. was supported in part by the Yukawa International Program for Quark-Hadron Sciences at Yukawa Institute for Theoretical Physics,

Kyoto University, Japan, and L.M. was supported through the Sofja Kovalevskaja program of the Alexander von Humboldt foundation. M.L. thanks A. Francis and O. Kaczmarek for interesting discussions concerning the prospects for lattice simulations. 


\section{Appendix A. Details of the unresummed computation}

\section{A.1. Matsubara sums, Fourier transform, spectral function}

We illustrate here the steps needed for the determination of the spectral function with the help of one of the basic structures appearing in the analysis, the function $\mathcal{I}_{2}$ defined in eq. (3.26). It turns out that in the course of the computation the expression splits into several parts, some of which may contain poles for specific spatial momenta; although such poles cancel at the end, it is important to "regulate" the intermediate expressions consistently so that no finite pieces go amiss. A convenient way to accomplish this is to redefine $\mathcal{I}_{2}$ as

$$
\mathcal{I}_{2}(\tau) \equiv \lim _{\lambda \rightarrow 0} \varlimsup_{K, Q} \frac{k^{2} e^{i k_{n} \tau}}{\left[K^{2}+\lambda^{2}\right] Q^{2}(K-Q)^{2}} .
$$

We have considered another regularization as well, writing the denominator as $1 /\left\{K^{2}\left[Q^{2}+\right.\right.$ $\left.\left.\lambda^{2}\right]\left[(K-Q)^{2}+\lambda^{2}\right]\right\}$, and checked that this leads to the same result in the limit $\lambda \rightarrow 0$.

As a first step, we carry out the Matsubara sums over $k_{n}, q_{n}$. Defining

$$
E_{k} \equiv \sqrt{k^{2}+\lambda^{2}}, \quad E_{q} \equiv|\mathbf{q}|, \quad E_{k q} \equiv|\mathbf{k}-\mathbf{q}|
$$

the sums can be written as

$$
\begin{aligned}
T^{2} \sum_{k_{n}, q_{n}} & \frac{e^{i k_{n} \tau}}{\left(k_{n}^{2}+E_{k}^{2}\right)\left(q_{n}^{2}+E_{q}^{2}\right)\left[\left(k_{n}-q_{n}\right)^{2}+E_{k q}^{2}\right]} \\
& =T^{3} \sum_{k_{n}, q_{n}, r_{n}} \frac{\beta \delta_{r_{n}+k_{n}-q_{n}} e^{i k_{n} \tau}}{\left(k_{n}^{2}+E_{k}^{2}\right)\left(q_{n}^{2}+E_{q}^{2}\right)\left(r_{n}^{2}+E_{k q}^{2}\right)} \\
& =\int_{0}^{\beta} \mathrm{d} \sigma T^{3} \sum_{k_{n}, q_{n}, r_{n}} \frac{e^{i k_{n}(\tau+\sigma)}}{k_{n}^{2}+E_{k}^{2}} \frac{e^{-i q_{n} \sigma}}{q_{n}^{2}+E_{q}^{2}} \frac{e^{i r_{n} \sigma}}{r_{n}^{2}+E_{k q}^{2}},
\end{aligned}
$$

where in the last step we used a representation of the (periodic) Kronecker delta, $\beta \delta_{t_{n}}=$

$\int_{0}^{\beta} \mathrm{d} \sigma e^{i t_{n} \sigma}$. The sums have now factorized and can be carried out like in eq. (3.5); care must be taken just in order to transform the time arguments to the interval $(0, \beta)$, which can be achieved for

$$
\tilde{G}(\tau, E) \equiv T \sum_{q_{n}} \frac{e^{i q_{n} \tau}}{q_{n}^{2}+E^{2}}
$$

by making use of the symmetries

$$
\tilde{G}(-\tau, E)=\tilde{G}(\tau+m \beta, E)=\tilde{G}(\tau, E), \quad m \in \mathbb{Z} .
$$

Since $\tau, \sigma$ only appear inside exponential functions in the result (cf. eq. (3.5)), the integral over $\sigma$ as well as the subsequent Fourier transform (eq. (2.5)) are trivial. The discontinuity 
as in eq. (2.6) can finally be taken by first setting $\exp \left( \pm i \omega_{n} \beta\right)=1$ in order to remove terms growing exponentially at large $|\omega|$, and by subsequently making use of

$$
\operatorname{Im}\left[\frac{1}{i \omega_{n}+\sum_{i} E_{i}}\right]_{\omega_{n} \rightarrow-i\left[\omega+i 0^{+}\right]}=-\pi \delta\left(\omega+\sum_{i} E_{i}\right) .
$$

Note that by making use of this relation we are advised to replace all other fractions by principal values $\left(\frac{1}{x+i 0^{+}}=\mathbb{P}\left(\frac{1}{x}\right)-i \pi \delta(x)\right)$.

Let us stress that all terms (in this as well as in other master sum-integrals) contain $\omega_{n}$ exclusively within the structures of eq. (A.6), always with at least one non-zero $E_{i}$; therefore no "transport peaks" $\sim \omega \delta(\omega)$ arise in the analytic continuation. In addition, there are no $\omega_{n}$-independent terms, which would correspond to contact terms in configuration space.

Denoting the result of these steps by $\tilde{\mathcal{I}}_{2}(\omega)$, we obtain

$$
\begin{aligned}
& \tilde{\mathcal{I}}_{2}(\omega)=\lim _{\lambda \rightarrow 0} \int_{\mathbf{k}, \mathbf{q}} \frac{\pi k^{2}}{4 E_{q} E_{k q}}\left\{\mathbb{P}\left(\frac{1}{E_{k}^{2}-\omega^{2}}\right)\right. {\left[\delta\left(\omega-E_{q}-E_{k q}\right)\left(1+n_{\mathrm{B} 1}+n_{\mathrm{B} 2}\right)\right.} \\
&+\delta\left(\omega+E_{q}-E_{k q}\right)\left(n_{\mathrm{B} 1}-n_{\mathrm{B} 2}\right) \\
&+\delta\left(\omega-E_{q}+E_{k q}\right)\left(n_{\mathrm{B} 2}-n_{\mathrm{B} 1}\right) \\
&\left.-\delta\left(\omega+E_{q}+E_{k q}\right)\left(1+n_{\mathrm{B} 1}+n_{\mathrm{B} 2}\right)\right] \\
&+\frac{\delta\left(\omega-E_{k}\right)-\delta\left(\omega+E_{k}\right)}{2 E_{k}} \mathbb{P}\left[\frac{1}{E_{k}+E_{q}+E_{k q}}+\frac{1}{-E_{k}+E_{q}+E_{k q}}\right. \\
&-2 E_{k q} n_{\mathrm{B} 1}\left(\frac{1}{\left(E_{k}-E_{q}\right)^{2}-E_{k q}^{2}}+\frac{1}{\left(E_{k}+E_{q}\right)^{2}-E_{k q}^{2}}\right) \\
&\left.\left.-2 E_{q} n_{\mathrm{B} 2}\left(\frac{1}{\left(E_{k}-E_{k q}\right)^{2}-E_{q}^{2}}+\frac{1}{\left(E_{k}+E_{k q}\right)^{2}-E_{q}^{2}}\right)\right]\right\},
\end{aligned}
$$

where

$$
n_{\mathrm{B} 1} \equiv n_{\mathrm{B}}\left(E_{q}\right), \quad n_{\mathrm{B} 2} \equiv n_{\mathrm{B}}\left(E_{k q}\right) .
$$

The expression is obviously antisymmetric in $\omega \rightarrow-\omega$; in the following we restrict, without loss of generality, to $\omega>0$.

In eq. (A.7), two different qualitative structures can be observed. We refer to the first group, with the constraints $\delta\left(\omega-E_{q}-E_{k q}\right)$ etc, as "phase space integrals"; and to the second

group, with the constraint $\delta\left(\omega-E_{k}\right)$, as "factorized integrals". We now proceed to discuss these two groups separately. The analysis parallels that in appendix A of ref. [24].

\section{A.2. Phase space integrals}

The phase space integrals are ultraviolet finite so that we can set $D \rightarrow 4$ from the outset. We fix the $z$-axis in the direction of $\mathbf{k}$ and carry out the integral over the directions of $\mathbf{q}$ by 
changing integration variables:

$$
\int \frac{\mathrm{d}^{3} \mathbf{q}}{(2 \pi)^{3}} \rightarrow \frac{1}{4 \pi^{2} k} \int_{0}^{\infty} \mathrm{d} q q \int_{|k-q|}^{k+q} \mathrm{~d} E_{k q} E_{k q}
$$

For $\omega>0$, the following results are easily verified:

$$
\begin{aligned}
\int_{|k-q|}^{k+q} \mathrm{~d} E_{k q} \delta\left(\omega-E_{q}-E_{k q}\right) \phi\left(E_{k q}\right) & =\theta(\omega-k) \theta\left(\frac{\omega+k}{2}-q\right) \theta\left(q-\frac{\omega-k}{2}\right) \phi(\omega-q) \\
\int_{|k-q|}^{k+q} \mathrm{~d} E_{k q} \delta\left(\omega+E_{q}-E_{k q}\right) \phi\left(E_{k q}\right) & =\theta(k-\omega) \theta\left(q-\frac{k-\omega}{2}\right) \phi(q+\omega) \\
\int_{|k-q|}^{k+q} \mathrm{~d} E_{k q} \delta\left(\omega-E_{q}+E_{k q}\right) \phi\left(E_{k q}\right) & =\theta(k-\omega) \theta\left(q-\frac{k+\omega}{2}\right) \phi(q-\omega)
\end{aligned}
$$

where we also simplified the notation as $E_{q} \rightarrow q$.

To go further let us start by considering the temperature-independent "vacuum part", to be denoted with $\delta_{\mathrm{ps}, 0} \tilde{\mathcal{I}}_{2}$ :

$$
\delta_{\mathrm{ps}, 0} \tilde{\mathcal{I}}_{2}(\omega) \equiv \lim _{\lambda \rightarrow 0} \int \frac{\mathrm{d}^{3} \mathbf{k}}{(2 \pi)^{3}} \mathbb{P}\left(\frac{\pi k^{2}}{E_{k}^{2}-\omega^{2}}\right) \int \frac{\mathrm{d}^{3} \mathbf{q}}{(2 \pi)^{3}} \frac{\delta\left(\omega-E_{q}-E_{k q}\right)}{4 E_{q} E_{k q}} .
$$

Changing integration variables as in eq. (A.9) and making use of eq. (A.10), we get

$$
\begin{aligned}
\delta_{\mathrm{ps}, 0} \tilde{\mathcal{I}}_{2}(\omega) & =\lim _{\lambda \rightarrow 0} \frac{4 \pi}{(2 \pi)^{3}} \int_{0}^{\omega} \mathrm{d} k \mathbb{P}\left(\frac{\pi k^{3}}{k^{2}+\lambda^{2}-\omega^{2}}\right) \frac{1}{(4 \pi)^{2}} \int_{\frac{\omega-k}{2}}^{\frac{\omega+k}{2}} \mathrm{~d} q \\
& =\lim _{\lambda \rightarrow 0} \frac{1}{32 \pi^{3}} \int_{0}^{\omega} \mathrm{d} k \mathbb{P}\left(\frac{k^{4}}{k^{2}+\lambda^{2}-\omega^{2}}\right) \\
& =\lim _{\lambda \rightarrow 0} \frac{\omega^{3}}{(4 \pi)^{3}}\left(\ln \frac{\lambda^{2}}{4 \omega^{2}}+\frac{8}{3}\right) .
\end{aligned}
$$

We see how the phase space integration contains a divergence, originating from an "onshell" configuration $(k \approx \omega)$; this will be cancelled against a corresponding divergence in the factorized vacuum integral, eq. (A.25), such that in the complete result the limit $\lambda \rightarrow 0$ can be taken.

Considering the thermal part, the substitution $\mathbf{q} \rightarrow \mathbf{k}-\mathbf{q}$, i.e. $E_{q} \leftrightarrow E_{k q}$, allows us to remove some redundancy, and the integral becomes

$$
\begin{aligned}
\delta_{\mathrm{ps}, \mathrm{T}} & \tilde{\mathcal{I}}_{2}(\omega) \equiv \lim _{\lambda \rightarrow 0} \frac{4 \pi}{(2 \pi)^{3}} \int_{0}^{\infty} \mathrm{d} k \mathbb{P}\left[\frac{\pi k^{3}}{4\left(k^{2}+\lambda^{2}-\omega^{2}\right)}\right] \frac{1}{4 \pi^{2}} \int_{0}^{\infty} \mathrm{d} q \\
& \times \int_{|k-q|}^{k+q} \mathrm{~d} E_{k q}\left\{2 \delta\left(\omega-q-E_{k q}\right) n_{\mathrm{B}}(q)+2 \delta\left(\omega+q-E_{k q}\right)\left[n_{\mathrm{B}}(q)-n_{\mathrm{B}}\left(E_{k q}\right)\right]\right\} \\
& =\lim _{\lambda \rightarrow 0} \frac{1}{16 \pi^{3}} \int_{0}^{\infty} \mathrm{d} k \mathbb{P}\left(\frac{k^{3}}{k^{2}+\lambda^{2}-\omega^{2}}\right)\left\{\theta(k-\omega) \int_{\frac{k-\omega}{2}}^{\frac{k+\omega}{2}} \mathrm{~d} q+\theta(\omega-k) \int_{\frac{\omega-k}{2}}^{\frac{\omega+k}{2}} \mathrm{~d} q\right\} n_{\mathrm{B}}(q),
\end{aligned}
$$


where we made use of eqs. (A.9) - A.11), and subsequently shifted $q+\omega \rightarrow q$ in one of the integrals, in order to always have $q$ as the argument of the Bose distribution. At this point it is advantageous to change the order of integration; the integration area can be illustrated as

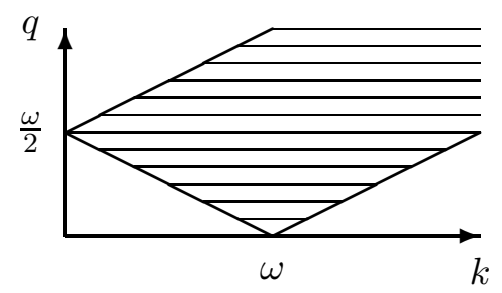

Thereby we obtain

$$
\begin{aligned}
\delta_{\mathrm{ps}, \mathrm{T}} \tilde{\mathcal{I}}_{2}(\omega) & =\lim _{\lambda \rightarrow 0} \frac{1}{16 \pi^{3}} \int_{0}^{\infty} \mathrm{d} q n_{\mathrm{B}}(q) \int_{|\omega-2 q|}^{\omega+2 q} \mathrm{~d} k \mathbb{P}\left(\frac{k^{3}}{k^{2}+\lambda^{2}-\omega^{2}}\right) \\
& =\frac{1}{16 \pi^{3}} \int_{0}^{\infty} \mathrm{d} q n_{\mathrm{B}}(q)\left[4 q \omega+\frac{\omega^{2}}{2} \ln \left|\frac{q+\omega}{q-\omega}\right|\right] .
\end{aligned}
$$

\section{A.3. Factorized spatial integrals}

The factorized integrals are more delicate than the phase space ones in the sense that the q-integral can contain ultraviolet divergences. In general this means that the integration measure is to be written as

$$
\int \frac{\mathrm{d}^{d} \mathbf{q}}{(2 \pi)^{d}} \rightarrow \frac{4}{(4 \pi)^{\frac{d+1}{2}} \Gamma\left(\frac{d-1}{2}\right)} \int_{0}^{\infty} \mathrm{d} q q^{d-1} \int_{-1}^{+1} \mathrm{~d} z\left(1-z^{2}\right)^{\frac{d-3}{2}},
$$

where $d \equiv D-1$ and $z=\mathbf{k} \cdot \mathbf{q} / k q$. If the integrand is independent of $z$, the $z$-integral yields

$$
\int_{-1}^{+1} \mathrm{~d} z\left(1-z^{2}\right)^{\frac{d-3}{2}}=\frac{\Gamma\left(\frac{1}{2}\right) \Gamma\left(\frac{d-1}{2}\right)}{\Gamma\left(\frac{d}{2}\right)} .
$$

If the q-integral is finite, as is always the case with thermal corrections, it is convenient to change integration variables like in eq. (A.9),

$$
\int_{-1}^{+1} \mathrm{~d} z \rightarrow \frac{1}{k q} \int_{|k-q|}^{k+q} \mathrm{~d} E_{k q} E_{k q} .
$$

Sometimes one is also in the lucky position that the q-integral can be directly identified with a known vacuum integral (see below).

Let us start by considering the temperature-independent "vacuum part", to be denoted by $\delta_{\mathrm{fz}, 0} \tilde{\mathcal{I}}_{2}$ :

$$
\begin{aligned}
\delta_{\mathrm{fz}, 0} \tilde{\mathcal{I}}_{2}(\omega) \equiv & \lim _{\lambda \rightarrow 0} \int \frac{\mathrm{d}^{d} \mathbf{k}}{(2 \pi)^{d}} \frac{\pi k^{2} \delta\left(\omega-E_{k}\right)}{2 E_{k}} \\
& \times \int \frac{\mathrm{d}^{d} \mathbf{q}}{(2 \pi)^{d}} \frac{1}{4 E_{q} E_{k q}} \mathbb{P}\left[\frac{1}{\omega+E_{q}+E_{k q}}+\frac{1}{-\omega+E_{q}+E_{k q}}\right] .
\end{aligned}
$$


Here we again restricted to $\omega>0$. Within the $\mathbf{k}$-integral the limit $\lambda \rightarrow 0$ can be immediately taken, so that $E_{k} \rightarrow k$; making use of eqs. (A.17), (A.18), we get

$$
\pi \int \frac{\mathrm{d}^{d} \mathbf{k}}{(2 \pi)^{d}} \frac{k \delta(\omega-k)}{2}=\frac{\omega^{3} \mu^{-2 \epsilon}}{4 \pi}\left[1+\epsilon\left(\ln \frac{\bar{\mu}^{2}}{4 \omega^{2}}+2\right)\right] .
$$

As far as the q-integral is concerned, we recall that one of the basic 1-loop integrals can, after integration over $q_{0}$, be written as

$$
\begin{aligned}
B_{0}\left(K^{2} ; 0,0\right) & \equiv \int \frac{\mathrm{d}^{D} Q}{(2 \pi)^{D}} \frac{1}{Q^{2}(K-Q)^{2}} \\
& =\int \frac{\mathrm{d}^{d} \mathbf{q}}{(2 \pi)^{d}} \frac{1}{4 E_{q} E_{k q}}\left[\frac{1}{i k_{0}+E_{q}+E_{k q}}+\frac{1}{-i k_{0}+E_{q}+E_{k q}}\right] .
\end{aligned}
$$

Therefore the 2 nd row of eq. (A.20) equals

$$
\operatorname{Re} B_{0}\left(-\omega^{2}+k^{2} ; 0,0\right)=\operatorname{Re} B_{0}\left(-\lambda^{2} ; 0,0\right)=\operatorname{Re}\left[\frac{\mu^{-2 \epsilon}}{(4 \pi)^{2}}\left(\frac{1}{\epsilon}+\ln \frac{\bar{\mu}^{2}}{-\lambda^{2}}+2\right)\right],
$$

where we made use of $\delta\left(\omega-E_{k}\right)$ in eq. (A.20) in order to write $\omega^{2}=k^{2}+\lambda^{2}$. In total, then,

$$
\delta_{\mathrm{fz}, 0} \tilde{\mathcal{I}}_{2}(\omega)=\lim _{\lambda \rightarrow 0} \frac{\omega^{3} \mu^{-4 \epsilon}}{(4 \pi)^{3}}\left(\frac{1}{\epsilon}+\ln \frac{\bar{\mu}^{2}}{4 \omega^{2}}+\ln \frac{\bar{\mu}^{2}}{\lambda^{2}}+4\right) .
$$

Here we observe again the significance of the intermediate regulator.

Summing together eqs. (A.14), A.25), logarithms of $\lambda$ cancel against each other, and we obtain the final vacuum part of $\tilde{\mathcal{I}}_{2}$, given on the first row of eq. (A.55).

As far as the thermal part is concerned, we change integration variables on the last row of eq. (A.7) so that the argument of $n_{\mathrm{B}}$ is always $E_{q}$. Furthermore, since the integral is exponentially convergent, we can use the measure in eq. (A.19). Partial fractioning the integrand and inserting $E_{k} \rightarrow \omega, E_{q} \rightarrow q$, we get

$$
\begin{aligned}
\delta_{\mathrm{fz}, \mathrm{T}} \tilde{\mathcal{I}}_{2}(\omega) & \equiv \lim _{\lambda \rightarrow 0} \frac{4 \pi}{(2 \pi)^{3}} \int_{0}^{\infty} \mathrm{d} k \frac{\pi k^{3} \delta\left(\omega-E_{k}\right)}{4 E_{k}} \frac{2 \pi}{(2 \pi)^{3}} \int_{0}^{\infty} \mathrm{d} q n_{\mathrm{B}}(q) \\
& \times \int_{|k-q|}^{k+q} \mathrm{~d} E_{k q} \mathbb{P}\left[\frac{1}{\omega-q+E_{k q}}-\frac{1}{\omega-q-E_{k q}}+\frac{1}{\omega+q+E_{k q}}-\frac{1}{\omega+q-E_{k q}}\right] .
\end{aligned}
$$

The integral over $E_{k q}$ is elementary, and sending $\lambda \rightarrow 0$, such that $\omega=\sqrt{k^{2}+\lambda^{2}} \rightarrow k$, it yields

$$
\lim _{\lambda \rightarrow 0} \ln \left|\frac{\left[(\omega-q)^{2}-(k+q)^{2}\right]\left[(\omega+q)^{2}-(k+q)^{2}\right]}{\left[(\omega-q)^{2}-(k-q)^{2}\right]\left[(\omega+q)^{2}-(k-q)^{2}\right]}\right|=\ln \left|\frac{q+\omega}{q-\omega}\right| .
$$

In total, then,

$$
\delta_{\mathrm{fz}, \mathrm{T}} \tilde{\mathcal{I}}_{2}(\omega)=\frac{\omega^{2}}{32 \pi^{3}} \int_{0}^{\infty} \mathrm{d} q n_{\mathrm{B}}(q) \ln \left|\frac{q+\omega}{q-\omega}\right| .
$$

Summing together eqs. (A.16), (A.28), we obtain the thermal part (second row) of eq. A.55). 


\section{A.4. An alternative derivation of the vacuum part}

While the method of sections A.1 A.3 works in all cases, the temperature-independent "vacuum parts" of the results can also be derived in a somewhat more straightforward manner. We illustrate this for the most complicated master integral appearing in our study, $\mathcal{I}_{5}(\tau)$, defined in eq. (3.18). The topologies from which this structure originates, already sketched in graph (j) of fig. 1, can be depicted somewhat more specifically as

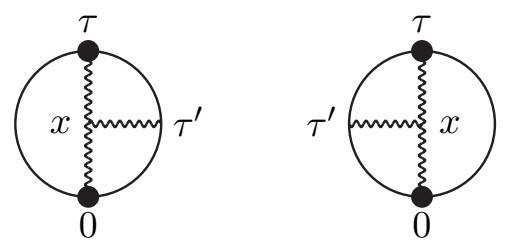

The idea of the approach is the following. Going to momentum space; carrying out several changes of integration variables; and ignoring possible problems with Matsubara zero modes, $q_{n}=0$ (this will be justified presently), the integrals over $x$ and $\tau^{\prime}$ can be carried out, and we end up with

$$
\begin{aligned}
\mathcal{I}_{5}(\tau)= & 2 \sum_{K} \frac{e^{i k_{n} \tau}}{K^{2}} £_{Q} \frac{1}{Q^{2}(K-Q)^{2}}\left\{(2 D-5) \mathbf{k}^{2}+(-3 D+5) k_{n}^{2}\right. \\
& \left.+\frac{2 k_{n}}{q_{n}}\left[Q^{2}+(D-2)\left(k_{n}^{2}-\mathbf{k}^{2}\right)\right]\right\} .
\end{aligned}
$$

We now replace the sum-integral $\oiint_{Q}$ through its zero-temperature limit, the $D$-dimensional integral $\int_{Q}$. In addition, the Fourier transform over $\tau$ and the subsequent analytic continuation and cut amount, due to the symmetry of the expression in $k_{n} \rightarrow-k_{n}$, to simply setting $k_{n} \rightarrow-i\left(\omega+i 0^{+}\right)$and taking the imaginary part. Thereby any dependence on the temperature disappears. Before the analytic continuation, it is useful to write $k_{n}^{2}=K^{2}-\mathbf{k}^{2}$.

The only complication is the handling of the second row of eq. A.30). Amusingly, the structure is similar to that met in connection with the so-called cyclic Wilson loop in ref. [25].3 We note, first of all, that in the zero-temperature limit the Matsubara zero mode plays no special role, and the apparent divergence $1 / q_{n}$ can be regulated by taking a principal value. Furthermore, introducing a Feynman parameter, the integrand can be written as

$$
\begin{aligned}
\frac{2 k_{n}}{q_{n}} \frac{1}{\left(k_{n}-q_{n}\right)^{2}+(\mathbf{k}-\mathbf{q})^{2}} & \rightarrow \frac{k_{n}}{q_{n}}\left[\frac{1}{\left(k_{n}-q_{n}\right)^{2}+(\mathbf{k}-\mathbf{q})^{2}}-\frac{1}{\left(k_{n}+q_{n}\right)^{2}+(\mathbf{k}-\mathbf{q})^{2}}\right] \\
& =\frac{4 k_{n}^{2}}{\left[\left(k_{n}-q_{n}\right)^{2}+(\mathbf{k}-\mathbf{q})^{2}\right]\left[\left(k_{n}+q_{n}\right)^{2}+(\mathbf{k}-\mathbf{q})^{2}\right]} \\
& =\int_{0}^{1} \mathrm{~d} s \frac{4 k_{n}^{2}}{\left\{(\mathbf{k}-\mathbf{q})^{2}+\left[(2 s-1) k_{n}-q_{n}\right]^{2}+4 s(1-s) k_{n}^{2}\right\}^{2}} .
\end{aligned}
$$

\footnotetext{
${ }^{3}$ In that case the analysis was originally carried out by M. Vepsäläinen.
} 
The discontinuity corresponding to eq. (A.30) can now be expressed as

$$
\begin{aligned}
\tilde{\mathcal{I}}_{5}(\omega)= & 2 \operatorname{Im} \int_{\mathbf{k}} \frac{1}{K^{2}}\left\{\int_{Q} \frac{1}{Q^{2}(K-Q)^{2}}\left[5(D-2) \mathbf{k}^{2}+(-3 D+5) K^{2}\right]\right. \\
& +4 k_{n}^{2} \int_{0}^{1} \mathrm{~d} s \int_{Q} \frac{1}{\left(Q^{2}+\tilde{M}^{2}\right)^{2}} \\
& \left.+4(D-2) k_{n}^{2}\left(k_{n}^{2}-\mathbf{k}^{2}\right) \int_{0}^{1} \mathrm{~d} s \int_{Q} \frac{1}{Q^{2}\left[(\tilde{K}-Q)^{2}+\tilde{M}^{2}\right]^{2}}\right\}_{i k_{n} \rightarrow \omega+i 0^{+}},
\end{aligned}
$$

where $\tilde{M}^{2} \equiv 4 s(1-s) k_{n}^{2}$ and $\tilde{K}^{2} \equiv(2 s-1)^{2} k_{n}^{2}+\mathbf{k}^{2}$.

The $Q$-integrals in eq. (A.32) are all familiar:

$$
\begin{aligned}
\int_{Q} \frac{1}{Q^{2}(K-Q)^{2}} & =\frac{\mu^{-2 \epsilon}}{(4 \pi)^{2}}\left(\frac{1}{\epsilon}+\ln \frac{\bar{\mu}^{2}}{K^{2}}+2\right), \\
\int_{Q} \frac{1}{\left(Q^{2}+\tilde{M}^{2}\right)^{2}} & =\frac{\mu^{-2 \epsilon}}{(4 \pi)^{2}}\left(\frac{1}{\epsilon}+\ln \frac{\bar{\mu}^{2}}{\tilde{M}^{2}}\right) \\
\int_{Q} \frac{1}{Q^{2}\left[(\tilde{K}-Q)^{2}+\tilde{M}^{2}\right]^{2}} & =\frac{\mu^{-2 \epsilon}}{(4 \pi)^{2} \tilde{K}^{2}} \ln \frac{\tilde{M}^{2}+\tilde{K}^{2}}{\tilde{M}^{2}} .
\end{aligned}
$$

In the middle case, the remaining integration over the Feynman parameter is also straightforward:

$$
\int_{0}^{1} \mathrm{~d} s \ln \frac{\bar{\mu}^{2}}{4 s(1-s) k_{n}^{2}}=\ln \frac{\bar{\mu}^{2}}{4 k_{n}^{2}}+2 .
$$

It remains to take the discontinuity and to carry out the integral over $\mathbf{k}$ as well as, where present, over $s$. For the structures on the first row of eq. (A.32) this leads directly to the "known" cases in eqs. (A.54), A.55). We discuss explicitly only the 2nd and 3rd rows of eq. (A.32), since these structures do not appear elsewhere.

Like in sections A.2, A.3, poles may appear in individual terms but are cancelled at the end; to handle these, we regulate the expression by replacing $K^{2} \rightarrow K^{2}+\lambda^{2}$ and denoting $E_{k}^{2} \equiv k^{2}+\lambda^{2}$. Inserting eqs. (A.34), (A.36), the structure on the 2 nd row of eq. (A.32) then becomes (omitting numerical coefficients)

$$
\delta_{2} \tilde{\mathcal{I}}_{5}(\omega) \equiv \frac{\mu^{-2 \epsilon}}{(4 \pi)^{2}} \int_{\mathbf{k}} \operatorname{Im}\left\{\frac{k_{n}^{2}}{k_{n}^{2}+E_{k}^{2}}\left(\frac{1}{\epsilon}+\ln \frac{\bar{\mu}^{2}}{4 k_{n}^{2}}+2\right)\right\}_{i k_{n} \rightarrow \omega+i 0^{+}} .
$$

We note that, for $\omega>0$,

$$
\begin{aligned}
\frac{\omega^{2}}{\left(-\omega+E_{k}-i 0^{+}\right)\left(\omega+E_{k}\right)} & =\mathbb{P}\left(\frac{\omega^{2}}{E_{k}^{2}-\omega^{2}}\right)+\frac{i \pi \omega}{2} \delta\left(\omega-E_{k}\right), \\
\frac{1}{\epsilon}+\ln \frac{\bar{\mu}^{2}}{4\left(\omega+i 0^{+}\right)\left(-\omega-i 0^{+}\right)}+2 & =\frac{1}{\epsilon}+\ln \frac{\bar{\mu}^{2}}{4 \omega^{2}}+2+i \pi .
\end{aligned}
$$


A non-zero contribution only arises by combining the imaginary part of eq. (A.38) with the real part of eq. (A.39), because the other possibility leads to

$$
\begin{aligned}
\int \frac{\mathrm{d}^{d} \mathbf{k}}{(2 \pi)^{d}} \mathbb{P}\left(\frac{1}{k^{2}+\lambda^{2}-\omega^{2}}\right) & =\int \frac{\mathrm{d}^{d} \mathbf{k}}{(2 \pi)^{d}} \frac{1}{k^{2}} \mathbb{P}\left(\frac{k^{2}+\lambda^{2}-\omega^{2}+\omega^{2}-\lambda^{2}}{k^{2}+\lambda^{2}-\omega^{2}}\right) \\
& =\frac{\sqrt{\omega^{2}-\lambda^{2}}}{4 \pi^{2}} \int_{0}^{\infty} \mathrm{d} k \mathbb{P}\left(\frac{1}{k-\sqrt{\omega^{2}-\lambda^{2}}}-\frac{1}{k+\sqrt{\omega^{2}-\lambda^{2}}}\right) \\
& =0 .
\end{aligned}
$$

Making use of eq. (A.21) in order to evaluate the $\mathbf{k}$-integral then leads to

$$
\delta_{2} \tilde{\mathcal{I}}_{5}(\omega)=-\frac{\omega^{3} \mu^{-4 \epsilon}}{(4 \pi)^{3}}\left[\frac{1}{\epsilon}+2\left(\ln \frac{\bar{\mu}^{2}}{4 \omega^{2}}+2\right)\right] .
$$

It remains to consider the structure on the 3rd row of eq. (A.32). Inserting eq. (A.35) and shifting the Feynman parameter by $\frac{1}{2}$ in order to make the integrand symmetric, it can be expressed as

$$
\delta_{3} \tilde{\mathcal{I}}_{5}(\omega) \equiv \frac{2 \mu^{-2 \epsilon}}{(4 \pi)^{2}} \int_{0}^{\frac{1}{2}} \mathrm{~d} s \int_{\mathbf{k}} \operatorname{Im}\left\{\frac{k_{n}^{2}}{k_{n}^{2}+E_{k}^{2}} \frac{k_{n}^{2}-k^{2}}{\left(2 k_{n} s\right)^{2}+k^{2}}\left(\ln \frac{k_{n}^{2}+k^{2}}{k_{n}^{2}}-\ln \left(1-4 s^{2}\right)\right)\right\}_{i k_{n} \rightarrow \omega+i 0^{+}} \text {(A.42) }
$$

The integrand is the product of three terms; the first one is like in eq. (A.38), while the 2nd and 3rd terms can for $\omega>0$ be written as

$$
\begin{aligned}
& \frac{\omega^{2}+k^{2}}{\left(-2 \omega s+k-i 0^{+}\right)(2 \omega s+k)}=\mathbb{P}\left(\frac{\omega^{2}+k^{2}}{k^{2}-4 \omega^{2} s^{2}}\right)+i \pi \theta(\omega-k) \delta(2 \omega s-k) \frac{\omega^{2}+k^{2}}{2 k}, \\
& \ln \frac{\left(-\omega+k-i 0^{+}\right)(\omega+k)}{\left(\omega+i 0^{+}\right)\left(-\omega-i 0^{+}\right)}-\ln \left(1-4 s^{2}\right)=\ln \frac{\left|k^{2}-\omega^{2}\right|}{\omega^{2}}-\ln \left(1-4 s^{2}\right)+i \pi \theta(k-\omega) .
\end{aligned}
$$

Various possible contributions can now be identified, according to whether the imaginary part comes from eq. (A.38), (A.43), or (A.44) (there is no contribution from multiplying the three imaginary parts, because of the appearances of $\theta(\omega-k)$ and $\theta(k-\omega)$ in eqs. (A.43), (A.44), respectively). Actually, the imaginary part of eq. (A.43) gives no contribution, because the real part of eq. A.44 vanishes for the value of $s$ that is allowed by the $\delta$-function of eq. (A.43). This then leaves two possibilities.

If we take the imaginary part from eq. (A.44) and write $\theta(k-\omega)=1-\theta(\omega-k)$, then the integral over unity can be reduced to integrals like that in eq. (A.40) and gives no contribution. A non-zero contribution comes from the integral (since the integral is UV-finite we set $d \rightarrow 3$ )

$$
\begin{aligned}
\delta_{3}^{\sqrt{\mathrm{A} .44}} \tilde{\mathcal{I}}_{5}(\omega) & \equiv-\frac{2 \pi}{(4 \pi)^{2}} \int_{0}^{\frac{1}{2}} \mathrm{~d} s \int_{0}^{\omega} \frac{\mathrm{d} k k^{2}}{2 \pi^{2}} \mathbb{P}\left(\frac{\omega^{2}}{E_{k}^{2}-\omega^{2}}\right) \mathbb{P}\left(\frac{\omega^{2}+k^{2}}{k^{2}-4 \omega^{2} s^{2}}\right) \\
& =\frac{\omega^{3}}{128 \pi^{3}} \int_{0}^{1} \mathrm{~d} x\left(1+x^{2}\right)\left[\frac{1}{x+1}+\mathbb{P}\left(\frac{1}{x-\sqrt{1-\lambda^{2} / \omega^{2}}}\right)\right] \ln \frac{1-x}{1+x},
\end{aligned}
$$


where we carried out the integration over $s$ and denoted $x \equiv k / \omega$; the two terms inside the square brackets come from the partial fractions of the first principal value (we have set $\lambda \rightarrow 0$ wherever the limit exists).

If we take the imaginary part from eq. (A.38), the integral becomes

$$
\delta_{3}^{\stackrel{(\mathrm{A.38}}{\mathcal{I}_{5}}} \tilde{\mathcal{I}}_{5}(\omega) \equiv \frac{\pi \omega}{(4 \pi)^{2}} \int_{0}^{\frac{1}{2}} \mathrm{~d} s \int_{0}^{\infty} \frac{\mathrm{d} k k^{2}}{2 \pi^{2}} \delta\left(\omega-E_{k}\right) \mathbb{P}\left(\frac{\omega^{2}+k^{2}}{k^{2}-4 \omega^{2} s^{2}}\right)\left[\ln \frac{\left|k^{2}-\omega^{2}\right|}{\omega^{2}}-\ln \left(1-4 s^{2}\right)\right]
$$

This time the integral over $k$ is trivial. Denoting $x=2 s$, a part of the $x$-integral can also be carried out, and we arrive at

$$
\delta_{3}^{\sqrt{\mathrm{A} .38}} \tilde{\mathcal{I}}_{5}(\omega)=\frac{\omega^{3}}{64 \pi^{3}}\left\{-\ln \frac{\lambda^{2}}{4 \omega^{2}} \ln \frac{\lambda^{2}}{\omega^{2}}-\int_{0}^{1} \mathrm{~d} x\left[\frac{1}{1+x}+\mathbb{P}\left(\frac{1}{\sqrt{1-\lambda^{2} / \omega^{2}}-x}\right)\right] \ln \left(1-x^{2}\right)\right\} .
$$

Adding up eqs. (A.45), (A.47), the coefficient of $\ln (1+x)$ develops a prefactor $x^{2}-1$; this cancels the pole (for $\lambda / \omega \rightarrow 0$ ) and leaves a regular finite integral. The coefficient of $\ln (1-x)$, in contrast, has a prefactor $x^{2}+3=4+2(x-1)+(x-1)^{2}$, so a singular integral remains. Denoting $y \equiv \sqrt{1-\lambda^{2} / \omega^{2}}-x$ and $\delta \equiv 1-\sqrt{1-\lambda^{2} / \omega^{2}} \approx \lambda^{2} / 2 \omega^{2}$, the singular integral can be written as

$$
\begin{aligned}
\delta_{3}^{\mathrm{s}} \tilde{\mathcal{I}}_{5}(\omega) & \equiv \frac{\omega^{3}}{128 \pi^{3}}\left\{-4 \int_{0}^{1} \mathrm{~d} x \mathbb{P}\left(\frac{1}{\sqrt{1-\lambda^{2} / \omega^{2}}-x}\right) \ln (1-x)\right\} \\
& =\frac{\omega^{3}}{128 \pi^{3}}\left\{-4\left[\int_{-\delta}^{+\delta} \mathrm{d} y+\int_{\delta}^{1-\delta} \mathrm{d} y\right] \mathbb{P}\left(\frac{1}{y}\right) \ln (y+\delta)\right\} \\
& =\frac{\omega^{3}}{128 \pi^{3}}\left\{-4 \int_{0}^{1} \frac{\mathrm{d} x}{x} \ln \frac{1+x}{1-x}-4 \int_{\delta}^{1} \frac{\mathrm{d} y}{y}\left[\ln \frac{y+\delta}{y}+\ln y\right]\right\},
\end{aligned}
$$

where in the first integral of the last row we wrote $x=y / \delta$ and in the second could set $\delta \rightarrow 0$ in the upper limit of the integration. Of the remaining integrals the singular one is now trivially integrated,

$$
-4 \int_{\delta}^{1} \frac{\mathrm{d} y}{y} \ln y=2 \ln ^{2}(\delta) \approx 2 \ln ^{2}\left(\frac{\lambda^{2}}{2 \omega^{2}}\right),
$$

whereas it requires a few more steps to show that

$$
\lim _{\delta \rightarrow 0}\left[-4 \int_{\delta}^{1} \frac{\mathrm{d} y}{y} \ln \frac{y+\delta}{y}\right]=-4 \int_{0}^{1} \frac{\mathrm{d} x}{x} \ln (1+x) .
$$

Summing up, all terms containing logarithms of $\lambda$ nicely cancel against each other, and we obtain

$$
\delta_{3} \tilde{\mathcal{I}}_{5}(\omega)=-\frac{\omega^{3}}{128 \pi^{3}}\left(2+\frac{4 \pi^{2}}{3}\right)
$$


We note that the peculiar $\pi^{2}$-terms, which appear nowhere else in our computation, can be traced back to the first term on the last row of eq. (A.48) as well as the term in eq. (A.50):

$$
\int_{0}^{1} \frac{\mathrm{d} x}{x} \ln \frac{(1+x)^{2}}{1-x}=\frac{\pi^{2}}{3} .
$$

Inserting finally the vacuum parts of eqs. (A.54), (A.55) as well as the newly determined (A.41), (A.51) into eq. (A.32), we obtain

$$
\tilde{\mathcal{I}}_{5}(\omega)=\frac{\omega^{3} \mu^{-4 \epsilon}}{16 \pi^{3}}\left(\frac{3}{\epsilon}+6 \ln \frac{\bar{\mu}^{2}}{4 \omega^{2}}+14-\frac{8 \pi^{2}}{3}\right) .
$$

This agrees with the vacuum part of eq. (A.58) which was independently determined with the method of sections A.1 A.3.

\section{A.5. Results for all independent sum-integrals}

We list here the results, after Fourier transform (eq. (2.5) ) and cut (eq. (2.6)), for the "master" structures of eqs. (3.18), (3.25)-(3.28). The bosonic and fermionic cases differ from each other simply by the exchange $n_{\mathrm{B}} \leftrightarrow-n_{\mathrm{F}}$; therefore, we only list the bosonic expressions here. The results read:

$$
\begin{gathered}
\tilde{\mathcal{I}}_{1}(\omega)=\frac{\omega^{3} \mu^{-4 \epsilon}}{16 \pi^{3}}\left(\frac{1}{6}\right)+\frac{1}{16 \pi^{3}} \int_{0}^{\infty} \mathrm{d} q n_{\mathrm{B}}(q)[4 q \omega] \\
\tilde{\mathcal{I}}_{2}(\omega)=\frac{\omega^{3} \mu^{-4 \epsilon}}{16 \pi^{3}}\left(\frac{1}{4 \epsilon}+\frac{1}{2} \ln \frac{\bar{\mu}^{2}}{4 \omega^{2}}+\frac{5}{3}\right) \\
+\frac{1}{16 \pi^{3}} \int_{0}^{\infty} \mathrm{d} q n_{\mathrm{B}}(q)\left[4 q \omega+\omega^{2} \ln \left|\frac{q+w}{q-w}\right|\right] \\
\tilde{\mathcal{I}}_{3}(\omega)=\frac{\omega^{3} \mu^{-4 \epsilon}}{16 \pi^{3}}\left(\frac{1}{24 \epsilon}+\frac{1}{12} \ln \frac{\bar{\mu}^{2}}{4 \omega^{2}}+\frac{2}{9}\right) \\
-\frac{1}{16 \pi^{3}} \int_{0}^{\infty} \mathrm{d} q n_{\mathrm{B}}(q)\left[q^{2} \ln \left|\frac{q+w}{q-w}\right|+q \omega \ln \frac{\left|q^{2}-\omega^{2}\right|}{\omega^{2}}\right] \\
\tilde{\mathcal{I}}_{4}(\omega)=\frac{\omega^{3} \mu^{-4 \epsilon}}{16 \pi^{3}}\left(\frac{1}{2 \epsilon}+\ln \frac{\bar{\mu}^{2}}{4 \omega^{2}}+\frac{23}{6}\right) \\
\quad-\frac{1}{16 \pi^{3}} \int_{0}^{\infty} \mathrm{d} q n_{\mathrm{B}}(q)\left[4 q \omega+\mathbb{P}\left(\frac{2 q \omega^{3}}{\omega^{2}-q^{2}}\right)\right]
\end{gathered}
$$




$$
\begin{aligned}
\tilde{\mathcal{I}}_{5}(\omega)= & \frac{\omega^{3} \mu^{-4 \epsilon}}{16 \pi^{3}}\left(\frac{3}{\epsilon}+6 \ln \frac{\bar{\mu}^{2}}{4 \omega^{2}}+14-\frac{8 \pi^{2}}{3}\right) \\
+\frac{1}{16 \pi^{3}} \int_{0}^{\infty} \mathrm{d} q n_{\mathrm{B}}(q) & \left\{24 q \omega+\mathbb{P}\left(\frac{8 q \omega^{3}}{\omega^{2}-q^{2}}\right)+20 \omega^{2} \ln \left|\frac{q+w}{q-w}\right|\right. \\
& \left.\quad+\frac{8 \omega^{4}}{q}\left[\frac{1}{q+\omega} \ln \frac{q+\omega}{\omega}+\mathbb{P}\left(\frac{1}{q-\omega} \ln \frac{\omega}{|q-\omega|}\right)\right]\right\} .
\end{aligned}
$$

Note that the integral $\int_{0}^{\infty} \mathrm{d} q q n_{\mathrm{B}}(q)$ appearing frequently could be carried out analytically (cf. eq. (4.12)), but many others cannot so we choose to display all thermal contributions in a uniform unintegrated form.

\section{Appendix B. Details of Hard Thermal Loop resummation}

We present here the details of the Hard Thermal Loop resummation, outlined and motivated in sec. 3.4. Concretely, the computations below amount to a derivation of eq. (4.3).

\section{B.1. Framework}

At $\omega \ll T$ the unresummed result of eq. (4.2) is logarithmically infrared divergent if we try to take the intercept determining the momentum diffusion coefficient $\kappa$ defined in eq. (2.7) (cf. eq. (4.14)). In order to obtain a result that is valid in this regime as well, the perturbative series needs to be reorganized. The well-known framework for achieving this is that of the Hard Thermal Loop (HTL) effective theory [17]. In order to avoid double-counting, we first need to subtract the unresummed version of the HTL contribution from the QCD result, whereby we should obtain a result that is infrared finite but not physical, since the contribution of the soft modes, $k \sim g T$, is not included (cf. eq. (2.9)); subsequently the resummed HTL contribution is added, whereby the result remains infrared finite and now involves the physical contribution of the Debye scale as well. For both purposes, we start by recalling the HTL form of the gluon self-energy in $D$ dimensions (at $\mathcal{O}\left(g^{4}\right)$ only the self-energy contribution is needed within the HTL theory, cf. section 3 of ref. [4]).

Carrying out the Matsubara sums over $q_{n}$ and $\left\{q_{n}\right\}$ in the self-energy of eq. (3.19); expanding the result to leading order in $i k_{n} / T, k / T$; and restricting for the moment to spatial components, one finds

$$
\Pi_{i j}(K)=g^{2}(D-2) \int_{\mathbf{q}} \frac{1}{q}\left[2 N_{\mathrm{f}} n_{\mathrm{F}}(q)+(D-2) N_{\mathrm{c}} n_{\mathrm{B}}(q)\right] \frac{v_{i} v_{j} i k_{n}}{i k_{n}-\mathbf{k} \cdot \mathbf{v}}+g^{2} \mathcal{O}\left(\frac{i k_{n}, k}{T}\right),
$$

where $\mathbf{v} \equiv \mathbf{q} / q$. Introducing the independent projection operators

$$
P_{\mu \nu}^{T}(K) \equiv \delta_{\mu i} \delta_{\nu j}\left(\delta_{i j}-\frac{k_{i} k_{j}}{k^{2}}\right), \quad P_{\mu \nu}^{E}(K) \equiv \delta_{\mu \nu}-\frac{K_{\mu} K_{\nu}}{K^{2}}-P_{\mu \nu}^{T}(K)
$$


and writing $\Pi_{i j}=P_{i j}^{T} \Pi_{T}+P_{i j}^{E} \Pi_{E}$, the scalar structures $\Pi_{T}, \Pi_{E}$ can be projected out:

$$
\begin{aligned}
& \Pi_{E}(K)=g^{2}(D-2) \frac{K^{2}}{k^{2}} \int_{\mathbf{q}} \frac{2 N_{\mathrm{f}} n_{\mathrm{F}}(q)+(D-2) N_{\mathrm{c}} n_{\mathrm{B}}(q)}{q} \frac{\mathbf{k} \cdot \mathbf{v}}{i k_{n}+\mathbf{k} \cdot \mathbf{v}}, \\
& \Pi_{T}(K)=g^{2} \int_{\mathbf{q}} \frac{2 N_{\mathrm{f}} n_{\mathrm{F}}(q)+(D-2) N_{\mathrm{c}} n_{\mathrm{B}}(q)}{q}\left(1-\frac{K^{2}}{k^{2}} \frac{\mathbf{k} \cdot \mathbf{v}}{i k_{n}+\mathbf{k} \cdot \mathbf{v}}\right) .
\end{aligned}
$$

For future reference, we also introduce the notation

$$
m_{\mathrm{E}}^{2} \equiv g^{2}(D-2) \int_{\mathbf{q}} \frac{1}{q}\left[2 N_{\mathrm{f}} n_{\mathrm{F}}(q)+(D-2) N_{\mathrm{c}} n_{\mathrm{B}}(q)\right]
$$

which for $D=4$ reproduces the known expression for the Debye mass parameter squared, given in eq. (4.4).

\section{B.2. Naive HTL analysis}

We first carry out an unresummed HTL computation, which needs to be subtracted from the naive QCD result in order to avoid double-counting and in order to obtain an infrared-finite result for the "matching coefficient" part of the final expression (cf. eq. (2.9)). This means that the self-energy is treated as an "insertion", i.e. as if it were an interaction. We also note that since the HTL theory only represents the infrared physics of QCD and has not been derived with relative accuracy $\mathcal{O}\left(g^{2}\right)$, the gauge coupling appearing in it is to be treated as the renormalized coupling. Then the unresummed result contains a free contribution like in eq. (3.9),

$$
\left[\rho_{\mathrm{E}}^{(2)}(\omega)\right]_{\mathrm{HTL}, \text { naive }}=\frac{g^{2} C_{F}}{6 \pi} \omega^{3},
$$

as well as a next-to-leading order term which we first write in configuration space,

$$
\left[G_{\mathrm{E}}^{(4)}(\tau)\right]_{\mathrm{HTL}, \text { naive }}=\frac{g^{2} C_{F}}{3} \oint_{K} \frac{e^{i k_{n} \tau}}{\left(K^{2}\right)^{2}}\left[(D-2) k_{n}^{2} \Pi_{T}(K)+K^{2} \Pi_{E}(K)\right] .
$$

To arrive at this expression we started from the form in eq. (3.20) and inserted $\Pi_{\mu \nu}=$ $P_{\mu \nu}^{T} \Pi_{T}+P_{\mu \nu}^{E} \Pi_{E}$. Plugging in $\Pi_{T}, \Pi_{E}$ from eqs. (B.3), (B.4) and partial fractioning the dependence on $i k_{n}$, we get

$$
\begin{gathered}
{\left[G_{\mathrm{E}}^{(4)}(\tau)\right]_{\mathrm{HTL}, \text { naive }}=\frac{g^{4} C_{F}(D-2)}{3} \int_{\mathbf{q}} \frac{2 N_{\mathrm{f}} n_{\mathrm{F}}(q)+(D-2) N_{\mathrm{C}} n_{\mathrm{B}}(q)}{q} \oint_{K} e^{i k_{n} \tau}\left[\frac{2}{K^{2}}-\frac{k^{2}}{\left(K^{2}\right)^{2}}\right.} \\
\left.\quad+\frac{1}{2(k-\mathbf{k} \cdot \mathbf{v})}\left(\frac{1}{i k_{n}+\mathbf{k} \cdot \mathbf{v}}-\frac{1}{i k_{n}+k}\right)-\frac{1}{2(k+\mathbf{k} \cdot \mathbf{v})}\left(\frac{1}{i k_{n}+\mathbf{k} \cdot \mathbf{v}}-\frac{1}{i k_{n}-k}\right)\right]
\end{gathered}
$$


It remains to Fourier transform (cf. eq. (2.5)) and take the discontinuity (cf. eq. (2.6)). For the structures at the end of the first row of eq. (B.8) this goes like before (cf. eq. (A.21) ) and yields, for $D \rightarrow 4$,

$$
\oiint_{K} \frac{e^{i k_{n} \tau}}{K^{2}} \rightarrow \frac{\omega}{4 \pi}, \quad \sum_{K} \frac{k^{2} e^{i k_{n} \tau}}{\left(K^{2}\right)^{2}} \rightarrow \frac{3 \omega}{8 \pi}, \quad \sum_{K} e^{i k_{n} \tau}\left[\frac{2}{K^{2}}-\frac{k^{2}}{\left(K^{2}\right)^{2}}\right] \rightarrow \frac{\omega}{8 \pi} \text {. }
$$

For the terms on the second row of eq. (B.8), on the other hand, the corresponding steps amount to

$$
T \sum_{k_{n}} \frac{e^{i k_{n} \tau}}{i k_{n}+\Delta} \rightarrow \pi \delta(\omega-\Delta)
$$

but the remaining integration over the spatial components of $\mathbf{k}$ requires regularization, so we have to proceed carefully.

Making use of the integration measure in eq. (A.17), save for $\mathbf{k}$, with the polar axis chosen in the direction of $\mathbf{v}=\mathbf{q} / q$ and denoting $z \equiv \mathbf{k} \cdot \mathbf{v} / k$, we are lead to consider the integral $(d \equiv D-1)$

$\mathcal{I}_{\mathrm{HTL}}(\omega) \equiv \int_{0}^{\infty} \mathrm{d} k \int_{-1}^{+1} \mathrm{~d} z \frac{k^{d-2}\left(1-z^{2}\right)^{\frac{d-3}{2}}}{2(4 \pi)^{\frac{d-1}{2}} \Gamma\left(\frac{d-1}{2}\right)}\left\{\frac{1}{1-z}[\delta(\omega-k z)-\delta(\omega-k)]-\frac{1}{1+z}[\delta(\omega-k z)]\right\}$,

where a term with $\delta(\omega+k)$ was omitted because of a restriction to $\omega>0$. In the term with $\delta(\omega-k)$ we can trivially integrate over $k$; in the terms with $\delta(\omega-k z)$ we can trivially integrate over $z$, noting that the constraints can get realized only for $k>\omega$. Renaming the integration variable subsequently as $k=\omega z, z>1$, and setting $d=3-2 \epsilon$, the result can be expressed as

$$
\mathcal{I}_{\text {HTL }}(\omega)=\frac{\omega^{1-2 \epsilon}}{2(4 \pi)^{1-\epsilon} \Gamma(1-\epsilon)}\left[\int_{1}^{\infty} \mathrm{d} z\left(z^{2}-1\right)^{-\epsilon}\left(\frac{1}{z-1}+\frac{1}{z+1}\right)-\int_{-1}^{+1} \mathrm{~d} z \frac{\left(1-z^{2}\right)^{-\epsilon}}{1-z}\right] .
$$

The first integral here can be rewritten as $\int_{1}^{\infty} \mathrm{d} y(y-1)^{-1-\epsilon}$ with $y=z^{2}$, and as an analytic function it vanishes. The second integral inside the square brackets evaluates to $1 / \epsilon-\ln 4+$ $\mathcal{O}(\epsilon)$. Expanding the prefactor as well and introducing the $\overline{\mathrm{MS}}$ scale parameter as before, we obtain

$$
\mathcal{I}_{\mathrm{HTL}}(\omega)=\frac{\omega \mu^{-2 \epsilon}}{8 \pi}\left(\frac{1}{\epsilon}+\ln \frac{\bar{\mu}^{2}}{4 \omega^{2}}\right) .
$$

Returning to eq. (B.8) and inserting $m_{\mathrm{E}}^{2}$ from eq. (B.5) as well as the simple structures from eq. (B.9), we finally arrive at

$$
\left[\rho_{\mathrm{E}}^{(4)}(\omega)\right]_{\mathrm{HTL}, \text { naive }}=\frac{g^{2} C_{F} m_{\mathrm{E}}^{2}}{3} \frac{\omega \mu^{-2 \epsilon}}{8 \pi}\left(\frac{1}{\epsilon}+\ln \frac{\bar{\mu}^{2}}{4 \omega^{2}}+1\right) .
$$




\section{B.3. Resummed intercept}

The remaining step is to carry out the resummed HTL computation (the last term in eq. (2.9)). As it happens, this problem was already addressed in ref. 44, albeit for the difference $\rho_{\mathrm{E}}(\omega) / \omega-\lim _{\omega \rightarrow 0} \rho_{\mathrm{E}}(\omega) / \omega$; the result is reproduced in eq. (4.6). In the following, it is then our task to consider the intercept $\lim _{\omega \rightarrow 0} \rho_{\mathrm{E}}(\omega) / \omega$, which was not addressed in ref. [4]. In the following we define $\left[\rho_{\mathrm{E}}\right]_{\mathrm{IR}}$ to be the spectral function computed within the HTL theory, but only up to linear order in a Taylor expansion in $\omega$ around zero, i.e. corresponding to the intercept $\lim _{\omega \rightarrow 0} \rho_{\mathrm{E}}(\omega) / \omega$. It turns out that this limit is logarithmically ultraviolet divergent within the HTL theory, with the same $1 / \epsilon$ as in eq. (B.14).

The computation proceeds somewhat analogously to the unresummed one above, only now eqs. (B.6), (B.7), as well as higher order corrections are resummed. Writing the expression after the Fourier transform in eq. (2.5), and making use of the evenness of $\Pi_{T}, \Pi_{E}$ in $k_{n} \rightarrow$ $-k_{n}$, we get

$$
\left[\tilde{G}_{\mathrm{E}}^{(4)}\left(\omega_{n}\right)\right]_{\mathrm{HTL}, \text { resum }}=-\frac{g^{2} C_{F}}{3} \int_{\mathbf{k}}\left[\frac{(D-2) \omega_{n}^{2}}{\omega_{n}^{2}+k^{2}+\Pi_{T}\left(\omega_{n}, \mathbf{k}\right)}+\frac{\omega_{n}^{2}+k^{2}}{\omega_{n}^{2}+k^{2}+\Pi_{E}\left(\omega_{n}, \mathbf{k}\right)}\right] .
$$

Taking now the discontinuity (eq. (2.6)) and noting that the first term of eq. (B.15) involves a high power of $\omega$, the contribution to the part linear in $\omega$ must come from the second term. Recalling eq. (B.3), we observe that $\omega_{n}^{2}+k^{2}$ nicely cancels out. For small $\omega$ we can furthermore write the structure inside $\Pi_{E}$ as

$$
\frac{\mathbf{k} \cdot \mathbf{v}}{-\omega+\mathbf{k} \cdot \mathbf{v}-i 0^{+}}=1+\mathbb{P}\left(\frac{\omega}{-\omega+\mathbf{k} \cdot \mathbf{v}}\right)+i \omega \pi \delta(\omega-\mathbf{k} \cdot \mathbf{v}) \approx 1+i \omega \pi \delta(\mathbf{k} \cdot \mathbf{v}),
$$

where in the real part the principal value integral leads to a contribution vanishing faster for $\omega \rightarrow 0$ than the unity. The unity leads directly to the $m_{\mathrm{E}}^{2}$ of eq. (B.5) and so we get

$$
\left[\rho_{\mathrm{E}}^{(4)}(\omega)\right]_{\mathrm{IR}, \text { resum }}=\frac{g^{4} C_{F}(D-2) \omega}{3} \int_{\mathbf{q}} \frac{2 N_{\mathrm{f}} n_{\mathrm{F}}(q)+(D-2) N_{\mathrm{c}} n_{\mathrm{B}}(q)}{q} \int_{\mathbf{k}} \frac{\pi k^{2} \delta(\mathbf{k} \cdot \mathbf{v})}{\left(k^{2}+m_{\mathrm{E}}^{2}\right)^{2}} .
$$

The $\mathbf{k}$-integral here can again be carried out by choosing $\mathbf{v}$ as the polar axis and making use of the measure in eq. (A.17):

$$
\begin{aligned}
\mathcal{I}_{\mathrm{IR}} & \equiv \pi \int \frac{\mathrm{d}^{d} \mathbf{k}}{(2 \pi)^{d}} \frac{k^{2} \delta(\mathbf{k} \cdot \mathbf{v})}{\left(k^{2}+m_{\mathrm{E}}^{2}\right)^{2}} \\
& =\frac{1}{(4 \pi)^{\frac{d-1}{2}} \Gamma\left(\frac{d-1}{2}\right)} \int_{0}^{\infty} \mathrm{d} k \frac{k^{d+1}}{\left(k^{2}+m_{\mathrm{E}}^{2}\right)^{2}} \int_{-1}^{+1} \mathrm{~d} z\left(1-z^{2}\right)^{\frac{d-3}{2}} \delta(k z) \\
& =\frac{m_{\mathrm{E}}^{-2 \epsilon}}{2(4 \pi)^{1-\epsilon} \Gamma(1-\epsilon)} \int_{0}^{\infty} \mathrm{d} y \frac{y^{1-\epsilon}}{(y+1)^{2}},
\end{aligned}
$$

where $y \equiv k^{2} / m_{\mathrm{E}}^{2}$. Expanding in $\epsilon$ we obtain

$$
\mathcal{I}_{\mathrm{IR}}=\frac{\mu^{-2 \epsilon}}{8 \pi}\left(\frac{1}{\epsilon}+\ln \frac{\bar{\mu}^{2}}{m_{\mathrm{E}}^{2}}-1\right),
$$


and eq. (B.17) in combination with eq. (B.5) then yields

$$
\left[\rho_{\mathrm{E}}^{(4)}(\omega)\right]_{\mathrm{IR}, \text { resum }}=\frac{g^{2} C_{F} m_{\mathrm{E}}^{2}}{3} \frac{\omega \mu^{-2 \epsilon}}{8 \pi}\left(\frac{1}{\epsilon}+\ln \frac{\bar{\mu}^{2}}{m_{\mathrm{E}}^{2}}-1\right) .
$$

Combining (as dictated by eq. (2.9)) the unresummed HTL result (eqs. (B.6), (B.14)) with the resummed IR limit (eq. (B.20) ) we arrive at eq. (4.3), from which $1 / \epsilon$-divergences have duly cancelled.

\section{B.4. Resummed $\omega$-dependence}

The remaining ingredient, the HTL-resummed contribution beyond the linear term in $\omega$, i.e.

$\left[\rho_{\mathrm{E}}(\omega)\right]_{\mathrm{HTL}, \text { resum }}-\left[\rho_{\mathrm{E}}(\omega)\right]_{\mathrm{IR} \text {, resum }}$, was determined in ref. [4], and is reproduced in eq. (4.6) of the main text. The four terms correspond to the transverse cut, electric cut, transverse pole, and electric pole, respectively. The $\hat{\omega}$-independent subtraction in the electric cut corresponds to eq. (B.17).

\section{References}

[1] G. Aarts, Transport and spectral functions in high-temperature QCD, PoS LAT2007 (2007) 001 [0710.0739].

[2] H.B. Meyer, Energy-momentum tensor correlators and viscosity, PoS LATTICE2008 (2008) 017 [0809.5202].

[3] J. Casalderrey-Solana and D. Teaney, Heavy quark diffusion in strongly coupled $\mathcal{N}=4$ Yang Mills, Phys. Rev. D 74 (2006) 085012 [hep-ph/0605199].

[4] S. Caron-Huot, M. Laine and G.D. Moore, A way to estimate the heavy quark thermalization rate from the lattice, JHEP 04 (2009) 053 [0901.1195].

[5] E. Braaten and M.H. Thoma, Energy loss of a heavy fermion in a hot plasma, Phys. Rev. D 44 (1991) 1298; Energy loss of a heavy quark in the quark - gluon plasma, Phys. Rev. D 44 (1991) 2625.

[6] B. Svetitsky, Diffusion of charmed quarks in the quark-gluon plasma, Phys. Rev. D 37 (1988) 2484.

[7] G.D. Moore and D. Teaney, How much do heavy quarks thermalize in a heavy ion collision?, Phys. Rev. C 71 (2005) 064904 hep-ph/0412346.

[8] S. Caron-Huot and G.D. Moore, Heavy quark diffusion in perturbative QCD at next-toleading order, Phys. Rev. Lett. 100 (2008) 052301 [0708.4232]; Heavy quark diffusion in $Q C D$ and $\mathcal{N}=4 S Y M$ at next-to-leading order, JHEP 02 (2008) 081 [0801.2173]. 
[9] C.P. Herzog, A. Karch, P. Kovtun, C. Kozcaz and L.G. Yaffe, Energy loss of a heavy quark moving through $\mathcal{N}=4$ supersymmetric Yang-Mills plasma, JHEP 07 (2006) 013 hep-th/0605158]; S.S. Gubser, Drag force in AdS/CFT, Phys. Rev. D 74 (2006) 126005 hep-th/0605182.

[10] S.S. Gubser, Momentum fluctuations of heavy quarks in the gauge-string duality, Nucl. Phys. B 790 (2008) 175 hep-th/0612143.

[11] M. Laine, G.D. Moore, O. Philipsen and M. Tassler, Heavy Quark Thermalization in Classical Lattice Gauge Theory: Lessons for Strongly-Coupled QCD, JHEP 05 (2009) 014 [0902.2856].

[12] Y. Akamatsu, T. Hatsuda and T. Hirano, Heavy Quark Diffusion with Relativistic Langevin Dynamics in the Quark-Gluon Fluid, Phys. Rev. C 79 (2009) 054907 [0809.1499]; R.S. Bhalerao and S. Gupta, Relativistic diffusion and heavy-ion collisions, Phys. Rev. C 79 (2009) 064901 [0901.4677]; A. Beraudo, A. De Pace, W.M. Alberico and A. Molinari, Transport properties and Langevin dynamics of heavy quarks and quarkonia in the Quark Gluon Plasma, Nucl. Phys. A 831 (2009) 59 [0902.0741]; R. Rapp and H. van Hees, Heavy Quarks in the Quark-Gluon Plasma, 0903.1096.

[13] B.I. Abelev et al. [STAR Collaboration], Transverse momentum and centrality dependence of high-p $p_{T}$ non-photonic electron suppression in $A u+A u$ collisions at $\sqrt{s_{N N}}=200$ GeV, Phys. Rev. Lett. 98 (2007) 192301 nucl-ex/0607012]; A. Adare et al. [PHENIX Collaboration], Energy Loss and Flow of Heavy Quarks in Au+Au Collisions at $\sqrt{s_{N N}}$ = 200 GeV, Phys. Rev. Lett. 98 (2007) 172301 nucl-ex/0611018.

[14] U. Gürsoy, E. Kiritsis, G. Michalogiorgakis and F. Nitti, Thermal Transport and Drag Force in Improved Holographic QCD, JHEP 12 (2009) 056 [0906.1890].

[15] R. Baier, B. Pire and D. Schiff, Dilepton production at finite temperature: Perturbative treatment at order $\alpha_{s}$, Phys. Rev. D 38 (1988) 2814; Y. Gabellini, T. Grandou and D. Poizat, Electron-positron annihilation in thermal QCD, Annals Phys. 202 (1990) 436; T. Altherr and P. Aurenche, Finite temperature QCD corrections to lepton-pair formation in a quark-gluon plasma, Z. Phys. C 45 (1989) 99.

[16] M. Asakawa, T. Hatsuda and Y. Nakahara, Maximum entropy analysis of the spectral functions in lattice QCD, Prog. Part. Nucl. Phys. 46 (2001) 459 hep-lat/0011040; G. Aarts, C. Allton, J. Foley, S. Hands and S. Kim, Spectral functions at small energies and the electrical conductivity in hot, quenched lattice QCD, Phys. Rev. Lett. 99 (2007) 022002 hep-lat/0703008; H.B. Meyer, A calculation of the shear viscosity in SU(3) gluodynamics, Phys. Rev. D 76 (2007) 101701 [0704.1801]; H.-T. Ding, O. Kaczmarek, 
F. Karsch, H. Satz and W. Söldner, Charmonium correlators and spectral functions at finite temperature, PoS LAT2009 (2009) 169 [0910.3098]; J. Engels and O. Vogt, Longitudinal and transverse spectral functions in the three-dimensional O(4) model, Nucl. Phys. B 832 (2010) 538 [0911.1939].

[17] R.D. Pisarski, Scattering Amplitudes in Hot Gauge Theories, Phys. Rev. Lett. 63 (1989) 1129; J. Frenkel and J.C. Taylor, High Temperature Limit of Thermal QCD, Nucl. Phys. B 334 (1990) 199; E. Braaten and R.D. Pisarski, Soft Amplitudes in Hot Gauge Theories: a General Analysis, Nucl. Phys. B 337 (1990) 569; J.C. Taylor and S.M.H. Wong, The Effective Action of Hard Thermal Loops in QCD, Nucl. Phys. B 346 (1990) 115.

[18] H.B. Meyer, Lattice Gauge Theory Sum Rule for the Shear Channel, 1005.2686.

[19] S. Caron-Huot, Asymptotics of thermal spectral functions, Phys. Rev. D 79 (2009) 125009 [0903.3958].

[20] K. Kajantie, M. Laine, K. Rummukainen and M. Shaposhnikov, $3 d S U(N)+$ adjoint Higgs theory and finite-temperature QCD, Nucl. Phys. B 503 (1997) 357 hep-ph/9704416.

[21] M. Laine and Y. Schröder, Two-loop QCD gauge coupling at high temperatures, JHEP 03 (2005) 067 hep-ph/0503061.

[22] I. Montvay and G. Münster, Quantum fields on a lattice (Cambridge University Press, Cambridge, 1994).

[23] D. Guazzini, H.B. Meyer and R. Sommer [ALPHA Collaboration], Non-perturbative renormalization of the chromo-magnetic operator in heavy quark effective theory and the $B^{*}-B$ mass splitting, JHEP 10 (2007) 081 [0705.1809].

[24] Y. Burnier, M. Laine and M. Vepsäläinen, Heavy quark medium polarization at next-toleading order, JHEP 02 (2009) 008 [0812.2105].

[25] Y. Burnier, M. Laine and M. Vepsäläinen, Dimensionally regularized Polyakov loop correlators in hot QCD, JHEP 01 (2010) 054 [0911.3480]. 